\title{
Predicting shear strength of CFS channels with slotted webs by machine learning models
}

\author{
Vitaliy V. Degtyarev ${ }^{1}$ (D)
}

Received: 5 April 2021 / Accepted: 24 June 2021 / Published online: 3 August 2021

(C) The Author(s), under exclusive licence to Springer Nature Switzerland AG 2021

\begin{abstract}
Staggered rectangular perforations (slots) are provided in the webs of cold-formed steel (CFS) beams and columns to reduce their thermal conductivity and improve the energy efficiency of CFS buildings. The perforations adversely affect the structural characteristics of the members, especially those governed by the web parameters, such as the shear strength and shear buckling. This paper presents machine learning (ML) models to predict the elastic shear buckling load and the ultimate shear strength of CFS channels with slotted webs. Support vector machine (SVM), decision tree (DT), random forest $(\mathrm{RF})$, and k-nearest neighbor $(\mathrm{KNN})$ regressors were trained using a large dataset of numerical results with 3512 samples. An extensive search was conducted to find optimal hyperparameters of the models that result in the best predictions and prevent overfitting. The models' performances were evaluated employing the ten-fold cross-validation method to make more data available for training and reduce bias and variance. The SVR, DT, and RF models demonstrate good prediction accuracy, which exceeds the accuracy of the existing descriptive equations. Relative feature importance was evaluated using the permutation and SHAP methods for each model. Partial dependence of the buckling load and the shear strength from the channel features was assessed. The predictions of the developed ML models were also compared with the predictions of previously developed artificial neural networks (ANNs). The comparisons demonstrated that ANNs showed higher accuracy in predicting the elastic buckling load, whereas the SVR model provided the most accurate shear strength predictions.
\end{abstract}

Keywords Cold-formed steel (CFS) · Perforated web · Shear buckling · Shear capacity · Machine learning ·

Predictive models

\section{Introduction}

Cold-formed steel (CFS) framing is structurally efficient and cost-effective due to its advantages, such as high strength-to-weight ratio, durability, sustainability, resilience, and ease of handling, transportation, and installation. CFS, however, possesses a high thermal conductivity, which may affect the energy efficiency of CFS buildings and require special thermal insulation. It has been demonstrated that the thermal conductivity of CFS members can be substantially reduced by providing punched or cut staggered rectangular perforations (slots) in the members' webs $[1,2]$. However, the perforations are detrimental to the structural

Vitaliy V. Degtyarev

vitaliy.degtyarev@newmill.com;vitdegtyarev@yahoo.com

1 New Millennium Building Systems, LLC, 3700 Forest Dr. Suite 501, Columbia, SC 29204, USA characteristics of the members [3-11], especially those governed by the web properties, such as shear and web crippling capacities [12,13].

Degtyarev and Degtyareva conducted extensive numerical parametric studies of the elastic shear buckling load, $V_{c r}$, and the ultimate shear strength, $V_{n}$ of CFS channels with slotted webs [14-16] performed on high-fidelity finite element (FE) models validated against the available test results [17]. The studies shed light on the parameters that affect the elastic buckling load and the ultimate shear strength of the perforated channels. Descriptive equations for predicting $V_{c r}$ and $V_{n}$ of such channels were developed, and a database of the FE simulation results was created and made publicly available [18]. The results of the numerical parametric studies also showed that it is very difficult, if not impossible, to come up with simple empirical equations for predicting $V_{c r}$ and $V_{n}$ of CFS channels with staggered web perforations due to the complex nature of the problem and many factors affecting the elastic buckling load and the shear strength. In the absence of simple empirical and analytical equations, 
machine learning (ML) methods are deemed a promising option.

Artificial intelligence (AI) and ML are wide-ranging computer science branches that deal with developing machines with simulated human intelligence and data-based predictive models. AI and ML have been successfully deployed in many industries, including healthcare [19], finance and investments [20], autonomous vehicles [21], manufacturing [22], and others. Publications on AI and ML applications in civil/structural engineering span more than three decades [23, 24], and their number has increased exponentially in recent years [25-27]. However, the actual deployment of $\mathrm{AI}$ and ML in civil/structural engineering is rather limited compared with other industries, despite their benefits, such as automation with low computational cost and high prediction accuracy.

Several papers have been published on the applications of artificial neural networks (ANNs) for buckling analysis of beam-columns [28] and cellular steel beams [29], as well as for different aspects of CFS structures, including space structure optimization [30], web crippling strength prediction [31], elastic distortional buckling stress determination [32, 33], and rotation capacity prediction [34]. The majority of ML algorithms considered in this work were employed for structural concrete: support vector machines (SVM) were studied in [35-41], decision trees (DT) in [40, 42], random forest (RF) in [40, 42-45], and k-nearest neighbors $(\mathrm{KNN})$ in $[37,40,42,46]$. Steel and steel-concrete composite structures have also seen limited applications of SVM [47-49] and DT and KNN [50].

ANNs for predicting $V_{c r}$ and $V_{n}$ of CFS channels with slotted webs were previously developed by the author [51]. In this study, four ML regressors, including SVM, DT, RF, and $\mathrm{KNN}$, for predicting $V_{c r}$ and $V_{n}$ of CFS channels with slotted webs were evaluated and compared with the ANN predictions. As was demonstrated in the literature review, the considered ML algorithms have been successfully used predominantly for reinforced concrete structures but have been rarely applied to steel structures and never evaluated for predicting the shear strength of CFS channels with slotted webs. The paper first describes the dataset used for training and testing the ML models, followed by a review of the ML algorithms considered in the study. The following section describes the implementation of the models, details of their training, testing, and hyperparameter tuning, and presents the performance of the models with the optimal hyperparameters. Relative feature importance and partial dependence of $V_{c r}$ and $V_{n}$ are presented and discussed next. Comparisons of $V_{c r}$ and $V_{n}$ predicted by the developed ML models, the existing descriptive equations, and previously developed ANNs are also presented and discussed. The novelty of this work consists in the application of new ML algorithms, implemented in an open-source framework, for predicting the buckling load and the shear strength of CFS channels with slotted webs, which allow for rapid predictions with an accuracy that is close to that of nonlinear FE simulations and exceeds the accuracy of the existing descriptive equations. The presented relative feature importance and partial dependence plots explain how different parameters of the channels affect $V_{c r}$ and $V_{n}$, resulting in a better understanding of the channels' structural response and may help determine optimal perforation patterns of the channels.

\section{Dataset}

The dataset of the elastic shear buckling loads and the ultimate shear strengths of CFS channels with slotted webs [18] was used for training and testing the ML models. The dataset includes FE simulation results for 3512 different channels described in [14-16]. Dimensional parameters of the studied channels are presented in Fig. 1. The 14 independent variables in the dataset are channel depth, $D$; channel flange width, $B$; channel flange stiffener length, $B_{1}$; channel thickness, $t$; length of slots, $L_{s l}$; height of slots, $W_{s l}$; spacing of slots in the longitudinal direction, $S_{s l}$; spacing of slots in the transverse direction, $B_{s l}$; the number of perforated regions, $N$; the number of slot rows, $n$; yield stress of steel, $F_{y}$; type of boundary conditions: realistic and test setup (designated in the models with numbers 1 and 2 , respectively); the aspect ratio, $a / h$; and height of the longitudinal stiffener, $h_{s t}$. The dependent variables are $V_{c r}$ and $V_{n}$. Distributions of the dataset variables shown in Fig. 2 demonstrate that the channels in the dataset are representative of the CFS channels with slotted webs available on the market.

A correlation matrix for the dataset variables is presented in Fig. 3. It shows that channel thickness, $t$, has the highest positive correlation with $V_{c r}$ and $V_{n}$, which can be classified as moderate, with coefficients of correlation of 0.73 and 0.77 , respectively. All other independent variables are weakly correlated with $V_{c r}$ and $V_{n}$, with a coefficient of correlation not exceeding 0.31 . The correlation between the independent variables is weak, except for $D$ and $B$, and $h_{s t}$ and $N$, which demonstrate a moderate correlation characterized by coefficients of correlation of 0.65 and 0.54 , respectively. The correlation between $V_{c r}$ and $V_{n}$ is moderate, with a coefficient of correlation of 0.77 .

\section{Review of machine learning models}

Four popular ML regressors, including support vector regressor (SVR), DT, RF, and KNN, were evaluated with respect to their predicting abilities of $V_{c r}$ and 


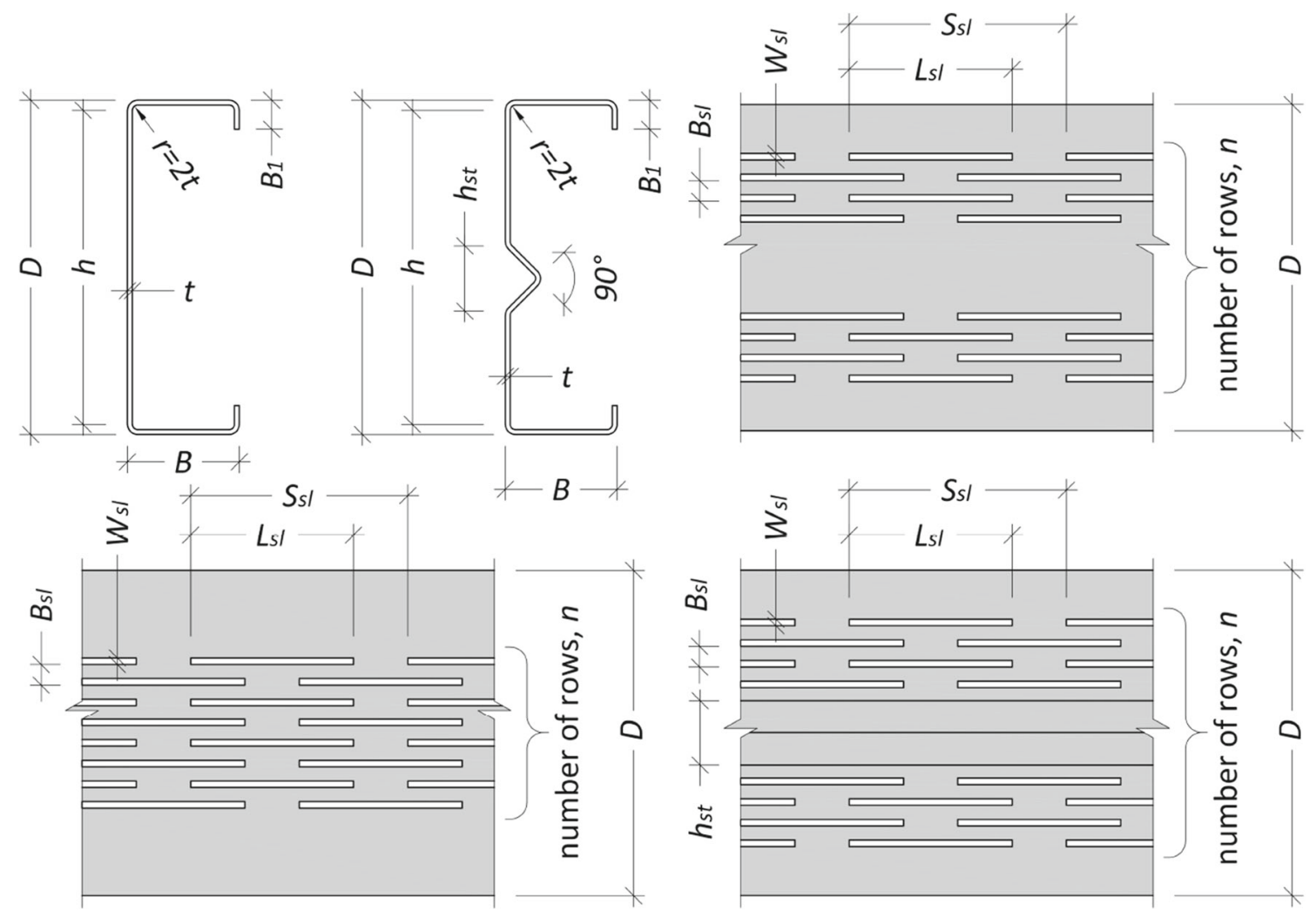

Fig. 1 Dimensional parameters of CFS channels with slotted webs
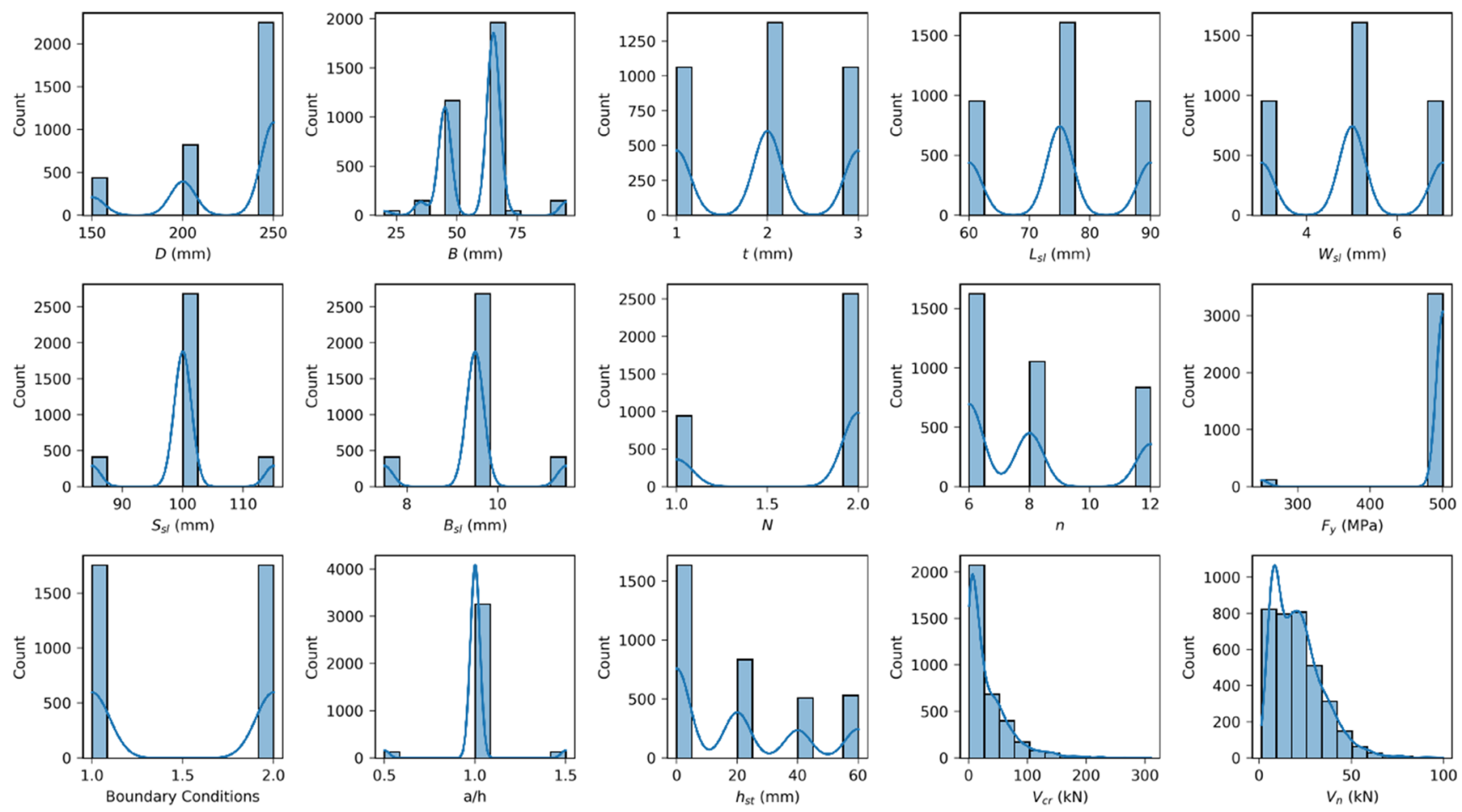

Fig. 2 Distributions of dataset variables 
Fig. 3 Correlation matrix of dataset variables

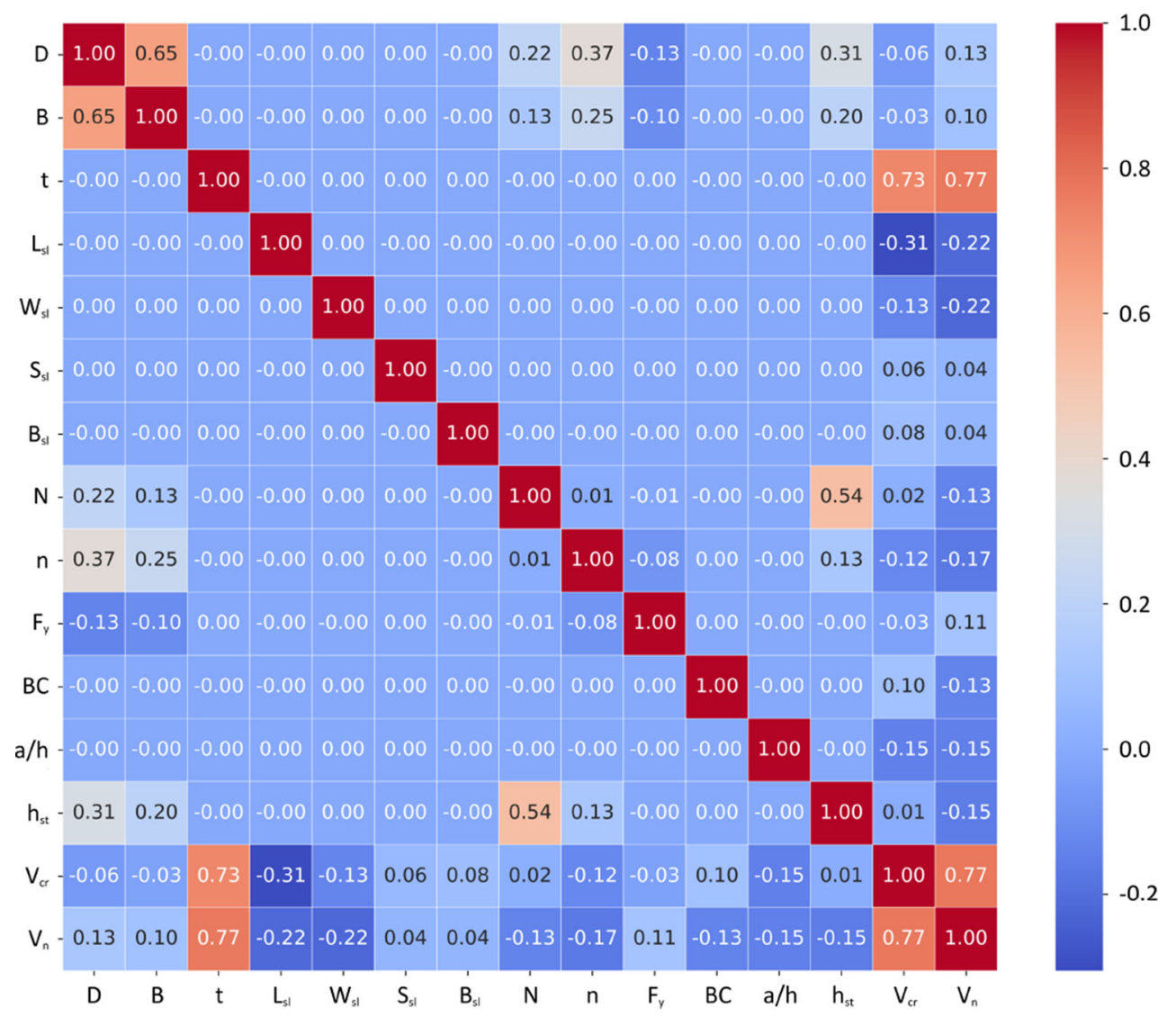

$V_{n}$ of CFS channels with slotted webs. All considered ML regressors are supervised algorithms, which learn by example using labeled training data. Figure 4 shows schematic representations of the models for predicting $V_{c r}$ and $V_{n}$. The models consist of input parameters (or features), ML algorithms (SVR, DT, RF, or KNN), and output parameters $\left(V_{c r}\right.$ and $\left.V_{n}\right)$. The input parameters are fed into the ML algorithm, which produces the output value. ML algorithms are trained from training data and finetuned by finding optimal hyperparameters, which are the algorithm parameters specified before training to control learning. Each ML algorithm has its own hyperparameters, as described in the following subsections. The input parameters of the models for predicting $V_{c r}$ and $V_{n}$ were $D, B, t, L_{s l}, W_{s l}, S_{s l}, B_{s l}, N, n$, boundary conditions (BC), $a / h, h_{s t}$, and $F_{y}$ (in the $V_{n}$ models only). The channel flange stiffener length, $B_{l}$, was excluded from the models' input parameters because it has an insignificant influence on $V_{c r}$ and $V_{n}$ [51]. The ML algorithms considered in this study are briefly described in the following subsections. More information about them and their practical implementation can be found in [52] and [53].

\section{Support vector regressor}

SVR is a regression application of SVM developed by Vapnik et al. [54-56] with the structural risk minimization principle in the basis. The $\epsilon-\mathrm{SVR}$ algorithm [54, 57] used in this study can be illustrated by considering training data $\left\{\left(x_{1}, y_{1}\right), \ldots,\left(x_{n}, y_{n}\right)\right\} \subset \chi \times \mathbb{R}$, where $x_{i}$ and $y_{i}$ are input and output parameters, respectively, and $\chi$ denotes the space of the input patterns. The $\epsilon$-SVR algorithm's objective is to find a hyperplane function $f(x)$ that has maximum $\epsilon$ deviation from targets $y_{i}$ and minimum flatness. Errors

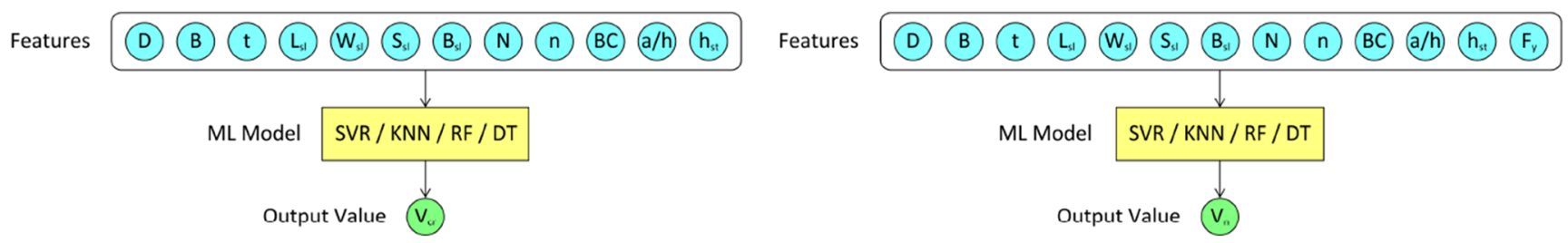

Fig. 4 Schematic representations of machine learning models for predicting $V_{c r}$ and $V_{n}$ 
smaller than $\epsilon$ are only acceptable in the model. The linear hyperplane function is described by Eq. 1 .

$$
f(x)=\langle w, x\rangle+b \text { with } w \in \chi, b \in \mathbb{R}
$$

where $\langle\cdot, \cdot\rangle$ represents the dot product in $\chi$.

To minimize the hyperplane flatness, a small $w$ needs to be found by minimizing the norm, i.e. $\|w\|^{2}=\langle w, w\rangle$ as follows.

minimize $\frac{1}{2}\|w\|^{2}$ subject to $\left\{\begin{array}{l}y_{i}-\left\langle w, x_{i}\right\rangle-b \leq \epsilon \\ \left\langle w, x_{i}\right\rangle+b-y_{i} \leq \epsilon\end{array}\right.$

Equation 2 is satisfied when a function $f(x)$ that approximates all pairs $\left(x_{i}, y_{i}\right)$ with $\epsilon$ precision can be found, which is not always possible. The concept of "soft margin" was introduced to allow for errors. It consists of using slack variables $\xi_{i}, \xi_{i}^{*}$ to give the algorithm flexibility in dealing with infeasible constraints. SVR with slack variables is formulated as follows:

$$
\begin{aligned}
& \operatorname{minimize} \frac{1}{2}\|w\|^{2}+C \sum_{i=1}^{n}\left(\xi_{i}+\xi_{i}^{*}\right) \\
& \text { subject to }\left\{\begin{array}{l}
y_{i}-\left\langle w, x_{i}\right\rangle-b \leq \epsilon+\xi_{i} \\
\left\langle w, x_{i}\right\rangle+b-y_{i} \leq \epsilon+\xi_{i}^{*} \\
\xi_{i}, \xi_{i}^{*} \geq 0
\end{array}\right.
\end{aligned}
$$

The "soft margin" constant $C$ controls the compromise between the flatness of $f(x)$ and the maximum tolerable deviations greater than $\epsilon$.

Nonlinear SVR is obtained by including nonlinear kernel functions into the algorithm. The nonlinear kernel function, which can be in the form of polynomial, radial basis (RBF), and sigmoid functions, transforms the original data into a high-dimensional feature space where a hyperplane function in the linear form can be found. The kernel function type and its parameters, the "soft margin" constant $C$, and the margin of tolerance $\epsilon$ are the SVR hyperparameters.

\section{Decision tree}

DT regression models have a tree structure that is incrementally developed by splitting the dataset into smaller subsets. The splitting occurs based on a series of questions determined by the DT algorithm. The learning begins at the root node, which includes all data and is split into two or more subsets. The splitting is repeated for the subsequentlevel nodes until a predefined limit (e.g., the maximum tree depth) is reached or when leaf nodes have only one sample. A sub-node that splits into further sub-nodes is called a decision node. Nodes that do not split are called leaves or terminal nodes.
There are various algorithms for growing a DT, which differ by the possible tree structure, the split finding criteria, the splitting stoppage criteria, and the model estimation within the leaf nodes. In this work, the classification and regression trees (CART) algorithm [52] implemented in scikit-learn [58] was used. The CART algorithm can be illustrated by considering a dataset $\left(x_{i}, y_{i}\right)$ for $i=$ $1,2, \ldots, N$, with $x_{i}=\left(x_{i 1}, x_{i 2}, \ldots, x_{i p}\right)$, where $x_{i}$ and $y_{i}$ are input and output parameters, $N$ is the number of samples, and $p$ is the number of input parameters. When the dataset is partitioned into $M$ regions $R_{1}, R_{2}, \ldots, R_{M}$, the model prediction in each region is a constant $c_{m}$ described by the following formula:

$f(x)=\sum_{m=1}^{M} c_{m} I\left(x \in R_{m}\right)$

where $I\left(x \in R_{m}\right)$ is the identity function that returns 1 if $x$ is in the subset $R_{m}$ and 0 otherwise.

When the criterion of minimization is in the form of the sum of squared errors $\sum\left(y_{i}-f\left(x_{i}\right)\right)^{2}$, the best $\hat{c}_{m}$ is the average of $y_{i}$ in region $R_{m}$ :

$\hat{c}_{m}=\operatorname{ave}\left(y_{i} \mid x_{i} \in R_{m}\right)$

The best binary partition in terms of minimum sum of squared errors is found using the following greedy algorithm. The pair of half-planes partitioned by a splitting variable $j$ and split point $s$ is defined as follows.

$R_{1}(j, s)=\left\{X \mid X_{j} \leq s\right\}$ and $R_{2}(j, s)=\left\{X \mid X_{j}>s\right\}$

The splitting variable $j$ and split point $s$ that solve Eq. 7 are sought.

$\min _{j, s}\left[\min _{c_{1}} \sum_{x_{i} \in R_{1}(j, s)}\left(y_{i}-c_{1}\right)^{2}+\min _{c_{2}} \sum_{x_{i} \in R_{2}(j, s)}\left(y_{i}-c_{2}\right)^{2}\right]$

The inner minimization is solved by

$\hat{c}_{1}=\operatorname{ave}\left(y_{i} \mid x_{i} \in R_{1}(j, s)\right)$ and $\hat{c}_{2}=\operatorname{ave}\left(y_{i} \mid x_{i} \in R_{2}(j, s)\right)$

After finding the best split, the dataset is partitioned into two resulting subsets, the splitting process for which is repeated. The process is repeated for the subsequent-level nodes. DT is prone to overfitting when the tree is very large $[59,60]$. Therefore, it is important for a DT to be small enough to avoid overfitting and large enough to capture the important relationships between the input and output parameters. The DT hyperparameters include the maximum depth of the tree, the minimum number of samples required to split an internal node, the minimum number of samples required to be at a leaf node, and others. 


\section{Random forest}

$\mathrm{RF}$ is an ensemble of DTs generally trained via the bootstrap aggregating (or bagging for short) method, which consists of training the same algorithm (DT) many times on different random subsets of the training set. In bagging, the sampling is performed with replacement, meaning that one sample may appear in different subsets. In the RF algorithm, multiple randomly generated DTs make independent predictions, which are averaged to obtain the final output value. Unlike DT, RF with multiple independent DTs is robust against overfitting.

The RF regression algorithm can be described as follows [52]:

1. For $b=1$ to $B$, where $b$ is an individual DT and $B$ is the total number of DTs (estimators):

(a) Draw a bootstrap sample of size $N$ from the training data.

(b) Grow a tree $T_{b}$ to the bootstrapped data by repeating the following steps for each node until the minimum node size or the maximum tree depth is reached:

(i) Randomly select $m$ variables from $p$ variables.

(ii) Find the best variable among $m$ and the best split point.

(iii) Split the node into two nodes.

2. Output the ensemble of trees $\left\{T_{b}\right\}_{1}^{B}$.

3. Make the final prediction as $\hat{f}_{R F}^{B}(x)=\frac{1}{B} \sum_{b=1}^{B} T_{b}(x)$.

The RF hyperparameters include those for DTs plus the number of trees in the forest.

\section{K-nearest neighbors}

KNN regression algorithm forecasts the output value by local interpolation of the $k$ nearest neighbors' output values in the training set. The number of neighbors $k$ is a hyperparameter set prior to training. The distance function used to define the distance between the neighbors can be expressed by the Minkowski metric, which is a generalization of the Euclidean and Manhattan distances:

$D(X, Y)=\left(\sum_{i=1}^{k}\left|x_{i}-y_{i}\right|^{p}\right)^{\frac{1}{p}}$

The Minkowski distance with the power parameter, $p$, being 1 and 2 corresponds to the Manhattan distance and the Euclidean distance, respectively.

The KNN regressor predicts output values by taking either an average (10) or an inverse distance weighted average (11) of the $k$ nearest neighbors with similar features. In the latter approach, closer neighbors have a more significant influence than the neighbors that are farther away.

$\hat{f}(x)=\frac{1}{k} \sum_{x_{i} \in N_{k}(x)} y_{i}$

$\hat{f}(x)=\frac{\sum_{x_{i} \in N_{k}(x)} \frac{1}{d_{i}} y_{i}}{\sum_{x_{i} \in N_{k}(x)} \frac{1}{d_{i}}}$

where $N_{k}(x)$ is the neighborhood of $x$ defined by the $k$ closest points $x_{i}$ in the training data, $d_{i}$ is the distance from the $i^{\text {th }}$ point to the estimated point.

The KNN hyperparameters are the number of neighbors, the weight function (uniform or inverse distance weighted), and the distance metric.

\section{Implementation and results}

The ML algorithms were implemented in scikit-learn [58], a Python-based open-source ML library. Optimal hyperparameters of the models were found through an extensive search, which was performed using Optunity [61] for SVR and the scikit-learn's GridSearch function for other models. Optunity is an open-source library that contains various optimizers for hyperparameter tuning. The Optunity's default particle swarm optimization [62] was used in this study. The GridSearch function conducts an undirected grid search by testing a predefined set of hyperparameter values.

The ML models were validated and tested employing the ten-fold cross-validation method, where the dataset is randomly divided into test and training data, which is further split into ten groups. The model is trained on nine groups of the training data and validated on the remaining group. The process is repeated ten times until each group has served as the validation set. The model's final performance is evaluated on the test data, which was not used for the model training. Such cross-validation method offers several benefits over the more traditional hold-out method, where the dataset is partitioned into training, validation, and test sets, with each set used for its purpose only. The benefits include the availability of more samples for the model training and avoiding the model dependence on a particular random choice of the samples selected for the training, validation, and test sets. Training models on multiple trainvalidation splits usually results in more accurate models with better generalization abilities. In this study, $80 \%$ of the dataset samples (2808) were used for training and $20 \%$ of the samples (704) for final testing.

The dataset features span varying degrees of magnitude and ranges (see Fig. 2), which may be an obstacle for 

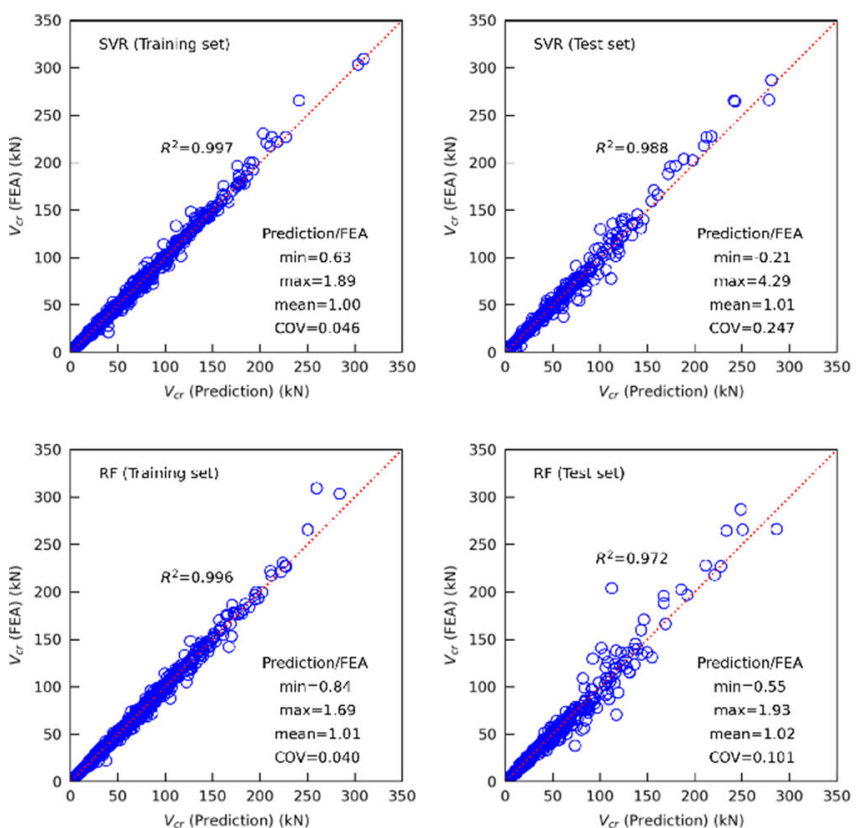

Fig. 5 Performance of the machine learning models for predicting $V_{c r}$

ML algorithms. To bring the input parameters to the same scale, each input parameter value in the training set was standardized using Eq. 12. The mean and standard deviation values obtained for the training set were used to standardize the test set's input values.

$x^{\prime}=\frac{x-\mu}{\sigma}$

where $x^{\prime}$ is the standardized value of the input parameter, $x$ is the original (non-standardized) value of the input
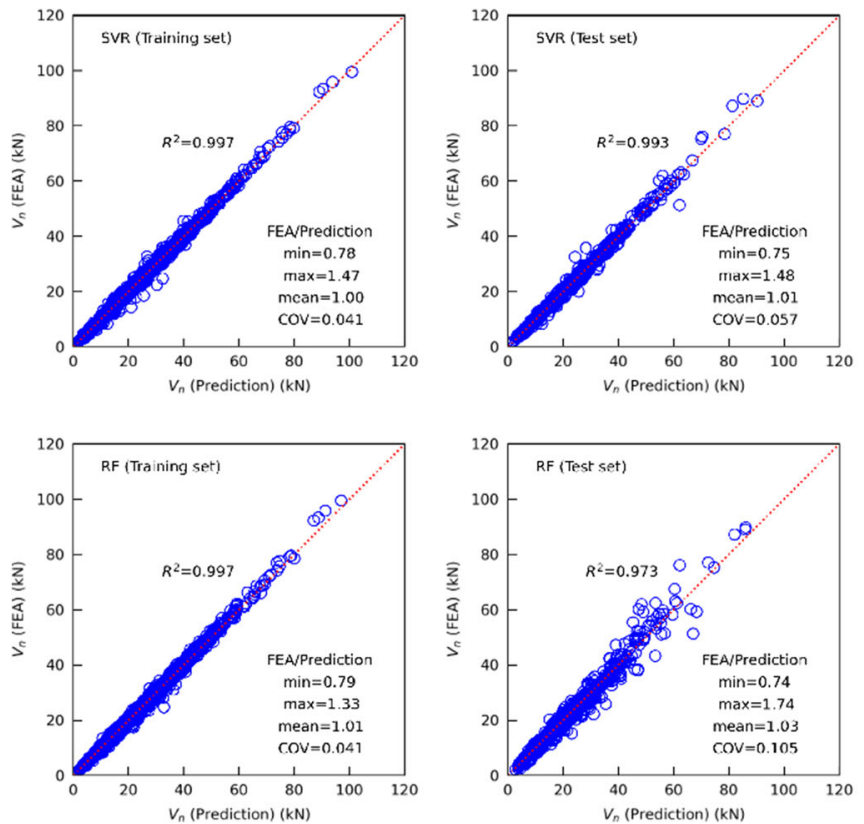
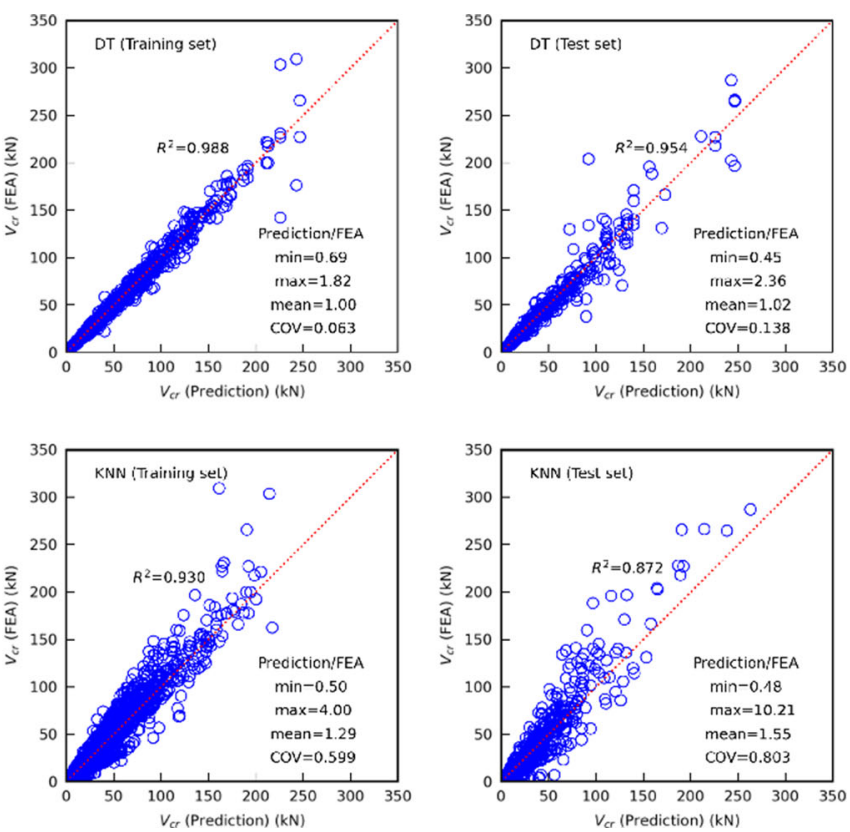

parameter, $\mu$ is the mean of the original values of the input parameter, and $\sigma$ is the standard deviation of the original values of the input parameter.

Mean squared error (MSE) values for the test set were used to evaluate the model performance and select the optimal hyperparameters.

$M S E=\frac{1}{n} \sum_{i=1}^{n}(y-\hat{y})^{2}$
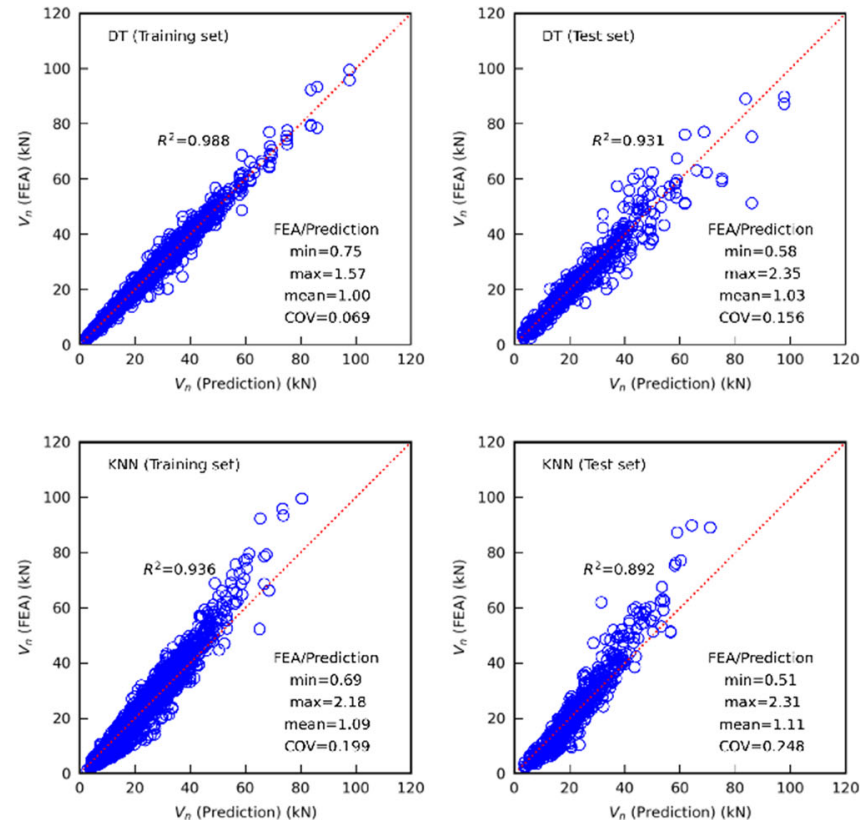

Fig. 6 Performance of the machine learning models for predicting $V_{n}$ 
Table 1 Performance metrics of optimized ML models for predicting $V_{c r}$

\begin{tabular}{|c|c|c|c|c|c|c|c|c|c|}
\hline \multirow[t]{2}{*}{ Model } & \multicolumn{3}{|c|}{$\operatorname{MSE}\left(\mathrm{kN}^{2}\right)$} & \multicolumn{3}{|c|}{ MAE $(\mathrm{kN})$} & \multicolumn{3}{|c|}{ MAPE (\%) } \\
\hline & Train & Test & All & Train & Test & All & Train & Test & All \\
\hline SVR & 3.78 & 20.39 & 7.16 & 0.63 & 2.05 & 0.93 & 1.83 & 10.35 & 3.57 \\
\hline DT & 16.58 & 80.57 & 29.38 & 1.46 & 3.61 & 1.89 & 4.28 & 9.49 & 5.33 \\
\hline $\mathrm{RF}$ & 4.84 & 49.09 & 13.70 & 0.90 & 2.77 & 1.27 & 2.79 & 7.07 & 3.65 \\
\hline KNN & 93.16 & 223.92 & 119.34 & 5.28 & 9.10 & 6.04 & 39.95 & 70.82 & 46.24 \\
\hline $\mathrm{ANN}^{1}$ & 3.85 & 20.08 & 7.11 & 1.25 & 2.42 & 1.49 & 11.63 & 16.87 & 12.68 \\
\hline $\mathrm{ANN}^{2}$ & 16.00 & 43.55 & 21.52 & 1.36 & 2.57 & 1.60 & 3.98 & 6.23 & 4.43 \\
\hline $\mathrm{ANN}^{3}$ & 2.86 & 13.73 & 5.04 & 0.60 & 1.64 & 0.81 & 4.64 & 7.98 & 5.31 \\
\hline $\mathrm{ANN}^{4}$ & 3.55 & 17.32 & 6.31 & 0.70 & 1.78 & 0.92 & 2.28 & 5.04 & 2.83 \\
\hline
\end{tabular}

${ }^{1} \mathrm{ANN}$ with one hidden layer and MSE loss function

${ }^{2}$ ANN with one hidden layer and MAPE loss function

${ }^{3}$ ANN with two hidden layers and MSE loss function

${ }^{4} \mathrm{ANN}$ with two hidden layers and MAPE loss function

where $n$ is the number of samples, $y$ is the output value, and $\hat{y}$ is the predicted output value.

Other common performance evaluation metrics [63] were also determined for the training and test sets, including mean absolute error (MAE), mean absolute percentage error (MAPE), the coefficient of determination $\left(R^{2}\right)$, the minimum, maximum, mean, and coefficient of variation values of the prediction-to-FEA ratios.

$M A E=\frac{1}{n} \sum_{i=1}^{n}|y-\hat{y}|$

$M A P E=\frac{100}{n} \sum_{i=1}^{n}\left|\frac{y-\hat{y}}{y}\right|$

$R^{2}=1-\frac{\sum_{i=1}^{n}(y-\hat{y})^{2}}{\sum_{i=1}^{n}(y-\bar{y})^{2}}$

where $\bar{y}$ is the mean of the $y$ values.
The optimal hyperparameters found for each ML model are listed below, with the scikit-learn's hyperparameter names shown in parentheses. Scikit-learn's default values were used for the hyperparameters not listed.

- SVR:

- kernel function (kernel): RBF for $V_{c r}$ and $V_{n}$,

- the "soft-margin" constant $C: 838867$ for $V_{c r}$ and 357047 for $V_{n}$,

- RBF's gamma (gamma): 0.07 for $V_{c r}$ and 0.03 for $V_{n}$,

- a margin of tolerance $\epsilon$ (epsilon): 2.16 for $V_{c r}$ and 0.69 for $V_{n}$.

- DT:

- the maximum depth of the tree (max_depth): None for $V_{c r}$ and $V_{n}$,

Table 2 Performance metrics of optimized ML models for predicting $V_{n}$

\begin{tabular}{|c|c|c|c|c|c|c|c|c|c|}
\hline \multirow[t]{2}{*}{ Model } & \multicolumn{3}{|c|}{$\operatorname{MSE}\left(\mathrm{kN}^{2}\right)$} & \multicolumn{3}{|c|}{ MAE $(\mathrm{kN})$} & \multicolumn{3}{|c|}{ MAPE (\%) } \\
\hline & Train & Test & All & Train & Test & All & Train & Test & All \\
\hline SVR & 0.55 & 1.52 & 0.75 & 0.40 & 0.73 & 0.46 & 2.10 & 3.72 & 2.43 \\
\hline DT & 2.12 & 14.38 & 4.57 & 0.91 & 2.22 & 1.17 & 4.83 & 10.95 & 6.06 \\
\hline $\mathrm{RF}$ & 0.61 & 5.62 & 1.61 & 0.53 & 1.47 & 0.71 & 2.92 & 7.41 & 3.82 \\
\hline KNN & 11.31 & 22.59 & 13.57 & 2.39 & 3.25 & 2.56 & 15.97 & 20.16 & 16.82 \\
\hline $\mathrm{ANN}^{1}$ & 0.54 & 1.68 & 0.77 & 0.51 & 0.84 & 0.57 & 3.23 & 5.05 & 3.60 \\
\hline $\mathrm{ANN}^{2}$ & 0.52 & 1.88 & 0.79 & 0.43 & 0.81 & 0.50 & 2.31 & 4.08 & 2.66 \\
\hline
\end{tabular}

${ }^{1} \mathrm{ANN}$ with one hidden layer and MSE loss function

${ }^{2} \mathrm{ANN}$ with one hidden layer and MAPE loss function 
- the minimum number of samples required to split an internal node (min_samples_split): 2 for $V_{c r}$ and $V_{n}$,

- the minimum number of samples at a leaf node (min_samples_leaf): 2 for $V_{c r}$ and $V_{n}$.

- RF:

- the number of trees in the forest (n_estimators): 500 for $V_{c r}$ and 100 for $V_{n}$,

- the maximum depth of the tree (max_depth): None for $V_{c r}$ and $V_{n}$,

- the minimum number of samples required to split an internal node (min_samples_split): 2 for $V_{c r}$ and $V_{n}$,

- the minimum number of samples at a leaf node (min_samples_leaf): 1 for $V_{c r}$ and $V_{n}$.

\section{- $\mathrm{KNN}$}

- the number of neighbors (n_neighbors): 3 for $V_{c r}$ and 5 for $V_{n}$,

- weight function (weights): uniform for $V_{c r}$ and $V_{n}$,

- the power parameter for the Minkowski metric (p): 1 for $V_{c r}$ and $V_{n}$.
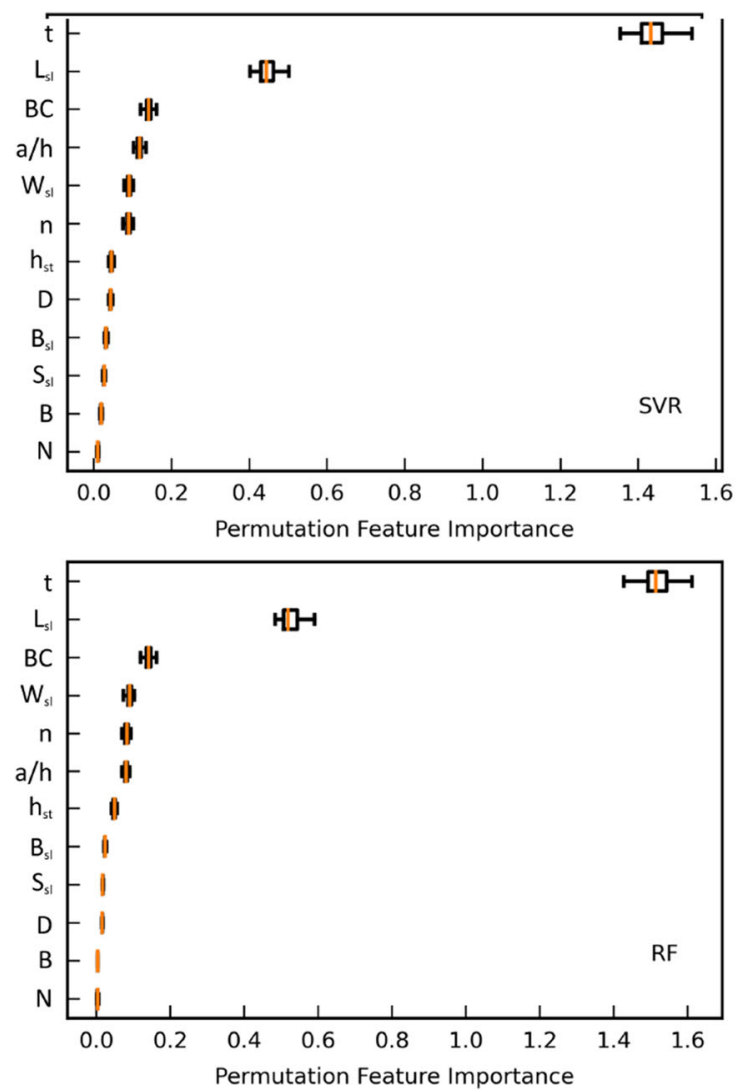

Performance of the developed ML models for predicting $V_{c r}$ and $V_{n}$ is presented in Figs. 5 and 6, respectively. The performance metrics are given in Figs. 5 and 6 and Tables 1 and 2.

Figure 5 and Table 1 show that the KNN regressor demonstrates considerably worse performance than SVR, DT, and RF, which predict $V_{c r}$ from the FE simulations reasonably well. SVR shows the best performance in terms of MSE and $R^{2}$ for the test set, but the SVR's coefficient of variation of the prediction-to-FEA ratios for the test set is considerably worse compared with RF and DT. It should also be noted that the SVR's minimum predictionto-FEA ratio is negative, and the maximum prediction-toFEA ratio is considerably greater than those for RF and DT. Therefore, it is concluded that RF demonstrates the best performance in predicting $V_{c r}$ among all evaluated models. SVR is the best model for predicting $V_{n}$ in terms of all performance metrics considered in the study. RF, DT, and KNN follow it. It should also be noted that all ML models predict $V_{n}$ significantly better than $V_{c r}$. The information presented in Figs. 5 and 6 and Tables 1 and 2 also indicate that the developed models possess good generalization ability, which is the ability to make good predictions of new data that have not been used in training.
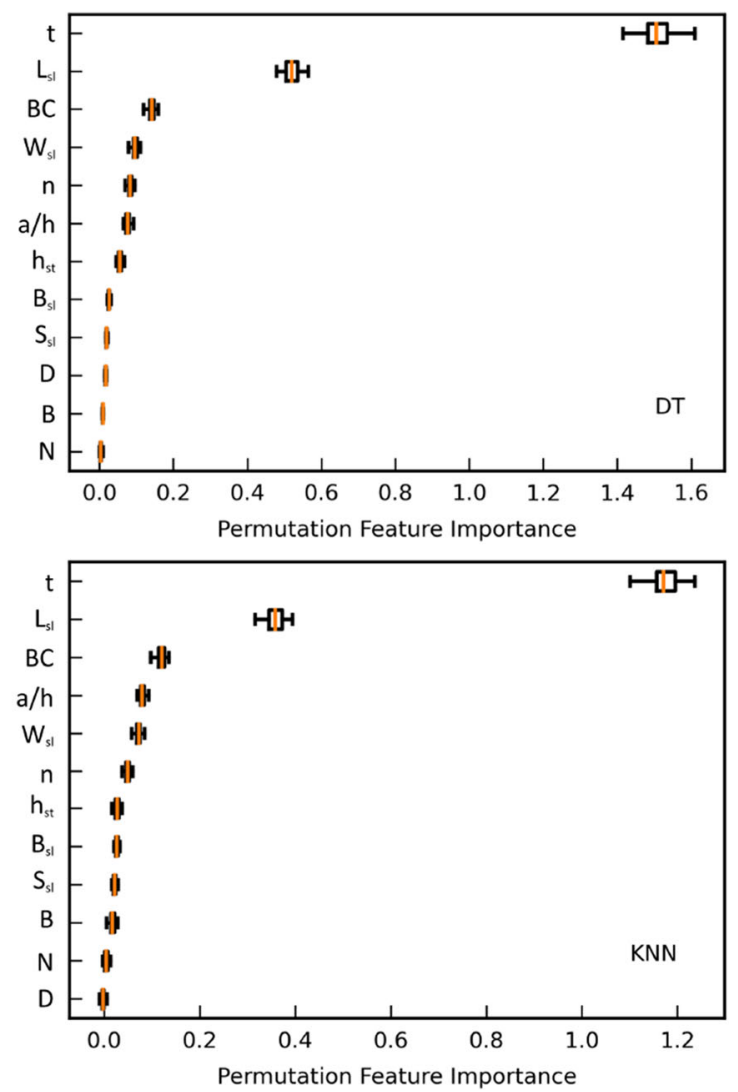

Fig. 7 Permutation feature importance of the machine learning models for predicting $V_{c r}$ 


\section{Relative feature importance and partial dependence}

To find out which property of the analyzed CFS channels with slotted webs has the most significant effect on the $V_{c r}$ and $V_{n}$ predictions, the relative feature importance was determined using permutation and SHAP methods. The permutation feature importance shows a reduction in the model score, usually in the form of $R^{2}$, when the feature is replaced with randomly permuted values. The model evaluation metric for the dataset modified by random shuffling of a single feature is determined and compared with the original dataset's evaluation metric. The process is repeated for each feature multiple times to obtain the mean and the standard deviation of the permutation importance score. The permutation feature importance, $i_{j}$ is computed as follows:

$i_{j}=s-\frac{1}{K} \sum_{k=1}^{K} s_{k, j}$

where subscript $j$ denotes each feature, $s$ is the model score for the original data with unpermuted features, $k$ is a permutation repetition, $K$ is the total number of the permutation repetitions, and $s_{k, j}$ is the model score for the data with the $j^{\text {th }}$ feature permuted at the $k^{\text {th }}$ repetition.

The permutation importance of the features in predicting $V_{c r}$ and $V_{n}$ is shown in Figs. 7 and 8, respectively. The evaluation metric (score) used in the permutation analysis was the coefficient of determination $R^{2}$. Therefore, a permutation feature importance value of 1 means that $R^{2}$ of the model reduced by 1 after the feature was permuted (see Eq. 17). The permutation feature importance values for $t$ are greater than one in Figs. 7 and 8, indicating that $R^{2}$ became negative for the data with permuted $t$. It means that, after shuffling, greater $t$ values corresponded to lower values of $V_{c r}$ and $V_{n}$, making the models trained on the original data (where $V_{c r}$ and $V_{n}$ increased when $t$ increased) unable to follow the trend of the permuted data. The permutation feature importance is model-dependent, which is reflected by a slightly different order of the relative feature importance values for the evaluated ML models observed in Figs. 7 and 8.

The channel thickness, $t$, has the most significant impact on $V_{c r}$. The channel thickness importance is considerably greater than the importance of all other features. The length of slots, $L_{S l}$, is the second most significant feature for predicting $V_{c r}$, followed by boundary conditions. The boundary conditions importance, however, is significantly
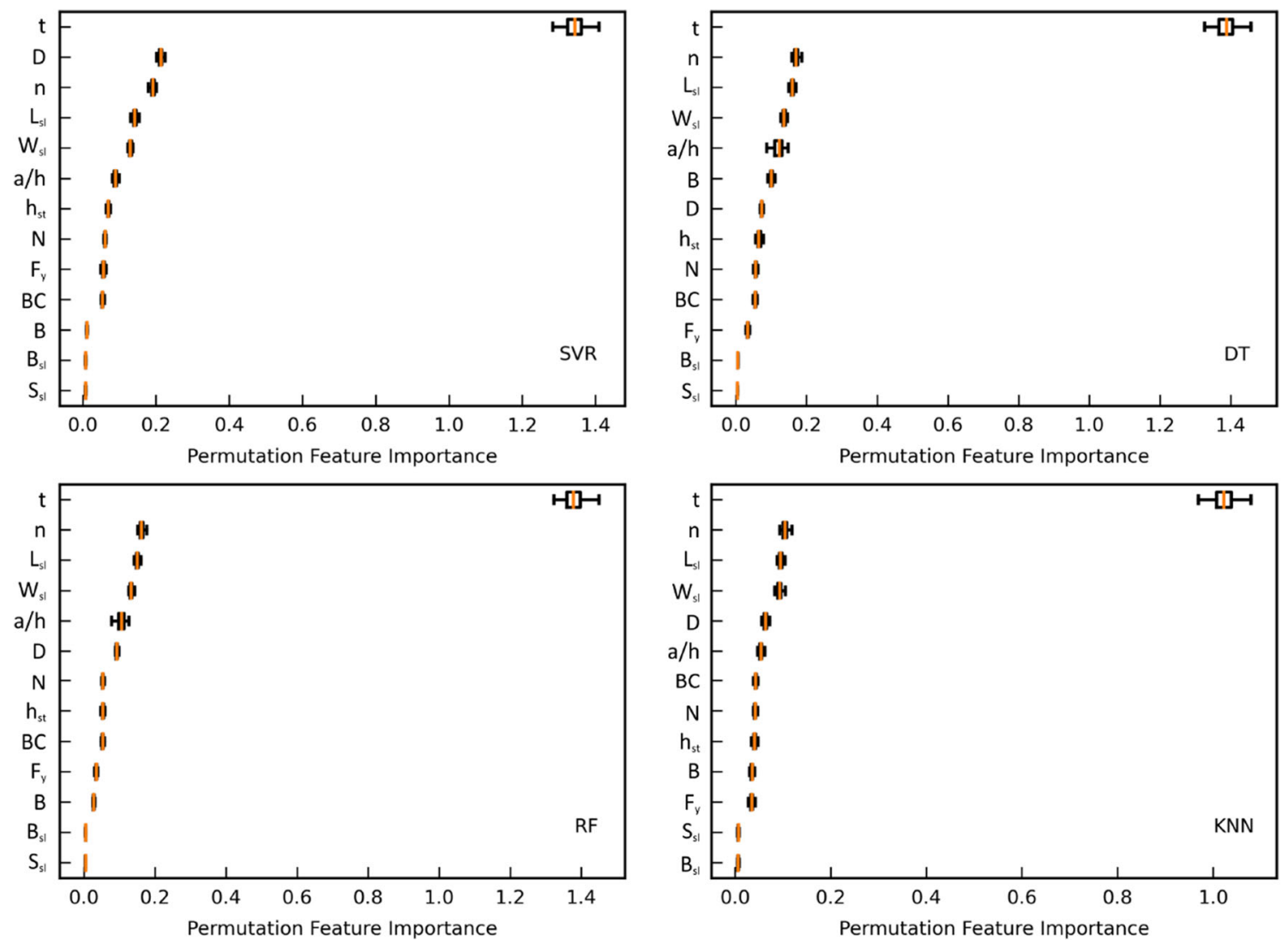

Fig. 8 Permutation feature importance of the machine learning models for predicting $V_{n}$ 
smaller than the importance of $L_{s l}$. All other features demonstrate even smaller permutation importance values for predicting $V_{c r}$, with the channel depth, $D$, the channel flange width, $B$, the number of perforated regions, $N$, and the spacing of slots in the longitudinal and transverse directions, $S_{s l}$ and $B_{s l}$ being at the bottom of the list.

In the models for predicting $V_{n}$, the channel thickness importance also significantly exceed the permutation importance of all other features. The importance of the channel depth, $D$, varies widely among the evaluated ML models, but in the most accurate model for predicting $V_{n}$, SVR, it is the second most important feature, followed by the number of slot rows, $n$, the length of slots, $L_{s l}$, the height of slots, $W_{s l}$, and the aspect ratio, $a / h$. The longitudinal stiffener height, $h_{s t}$, the number of perforated regions, $N$, the steel yield stress, $F_{y}$, and boundary conditions have even more minor effects on $V_{n}$ in the SVR model, with the importance of the remaining features (channel flange width,
$B$, and the spacing of slots in the longitudinal and transverse directions, $S_{s l}$ and $B_{s l}$ ) being close to zero.

The SHAP method, which stands for Shapley Additive Explanations [64], relies on the Shapley values from the cooperative game theory. The SHAP feature importance is estimated by comparing predictions for models with and without each feature. In contrast to the permutation feature importance, which is based on the decrease in model performance, SHAP is based on the magnitude of feature attributions. Figures 9 and 10 show the SHAP relative feature importance of the evaluated ML models for predicting $V_{c r}$ and $V_{n}$, respectively. In the models for predicting $V_{c r}$, the SHAP feature importance orders are similar to those from the permutation method discussed previously, except the channel depth, $D$, demonstrates slightly greater importance per the SHAP method. For the most accurate model for predicting $V_{n}$, SVR, the SHAP relative importance of the features is also similar
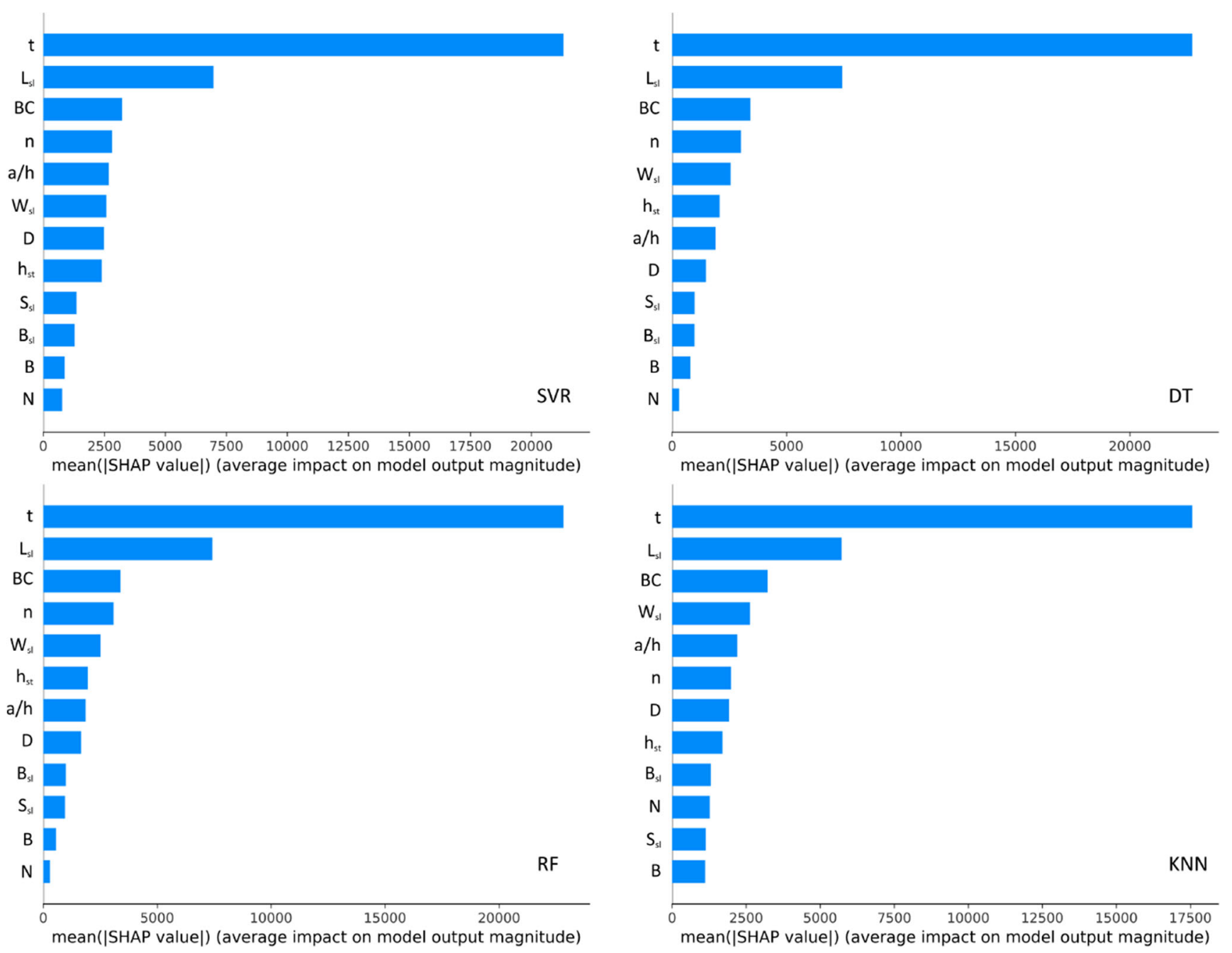

Fig. 9 SHAP feature importance of the machine learning models for predicting $V_{c r}$ 

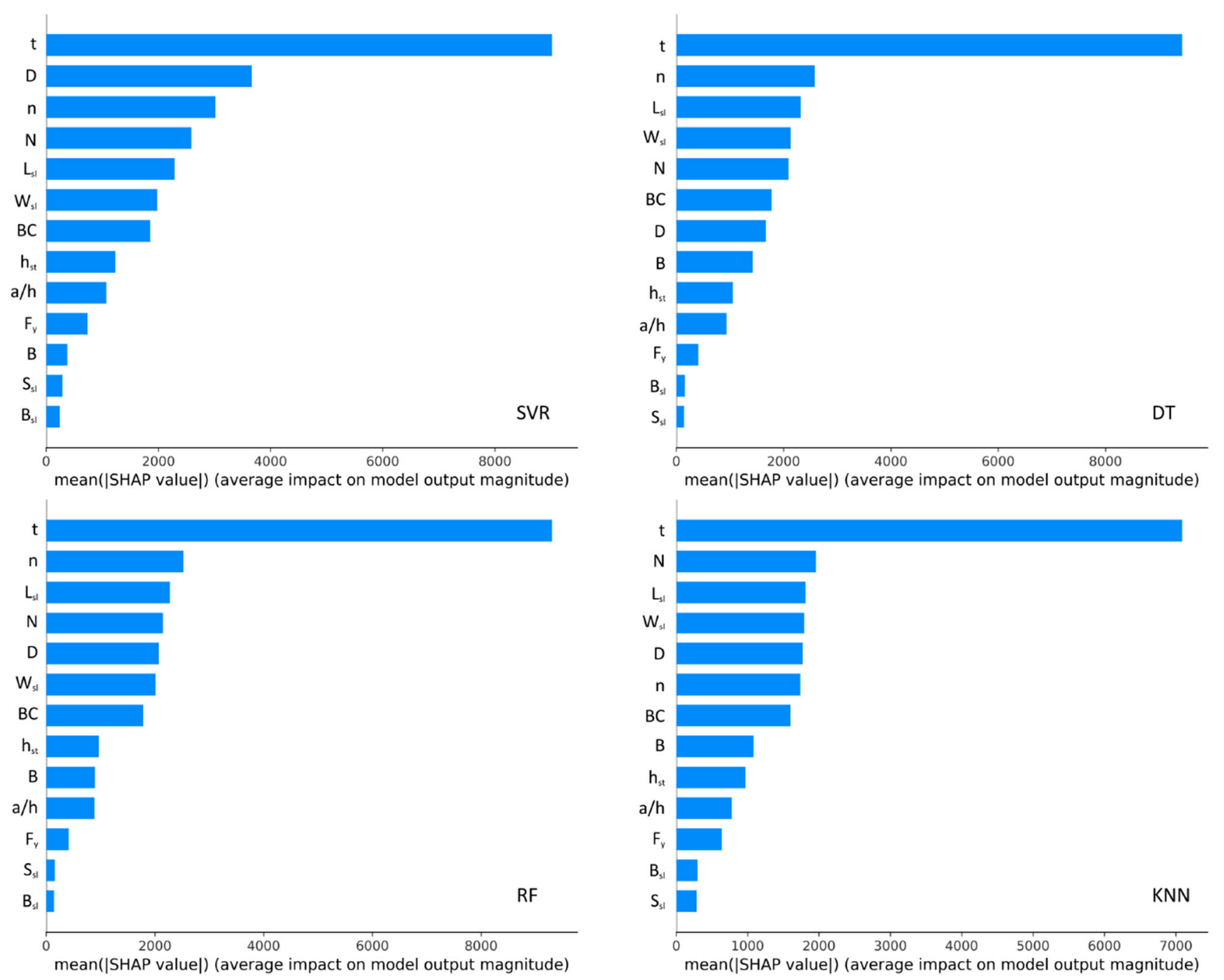

Fig. 10 SHAP feature importance of the machine learning models for predicting $V_{n}$

to the permutation feature importance, except the number of perforated regions, $N$, and boundary conditions show noticeably greater importance, while the aspect ratio, $a / h$, has smaller importance per the SHAP method.

The relative feature importance described above provides valuable information about how each feature affects the target, but it does not show the magnitude of the target change due to the change of each feature, which partial dependence plots can illustrate. Partial dependence plots demonstrate the effect of each feature on the predicted variable. They can reveal whether the relationships between the target and features are linear or nonlinear. Figures 11 and 12 show partial dependence plots for the developed ML models for predicting $V_{c r}$ and $V_{n}$. It can be seen from these figures that the shapes of the relationships between the features and the targets for different models are relatively similar. However, there are some differences in the effect magnitudes. An increase of the channel depth, $D$, results in a negligible reduction of $V_{c r}$ and a noticeable increase of $V_{n}$, especially for the SVR model, which is the most accurate in predicting $V_{n}$. An increase of the channel flange width, $B$, practically does not affect $V_{c r}$ and $V_{n}$. An increase of the channel thickness, $t$, causes significant increases in $V_{c r}$ and $V_{n}$. The relationship between $V_{n}$ and the channel thickness, $t$, within the considered range, is linear, whereas $V_{c r}$ shows a greater increase when $t$ changes from 2 to 3 $\mathrm{mm}$ than when it changes from 1 to $2 \mathrm{~mm}$. Both $V_{c r}$ and $V_{n}$ decrease when the length of slots, $L_{s l}$, increased. The relationship is nonlinear, with greater $V_{c r}$ and $V_{n}$ reductions occurring when $L_{s l}$ increases from 60 to $75 \mathrm{~mm}$ than when it changes from 75 to $90 \mathrm{~mm}$. The effects of the slot height, $W_{s l}$, on $V_{c r}$ and $V_{n}$ are linear: both values reduce when $W_{s l}$ increases. Increases in the spacing of slots in the longitudinal and transverse directions, $S_{s l}$ and $B_{s l}$, result in slight increases in $V_{c r}$ and practically no change in $V_{n} . V_{c r}$ is not affected by changes in the number of perforated regions, 

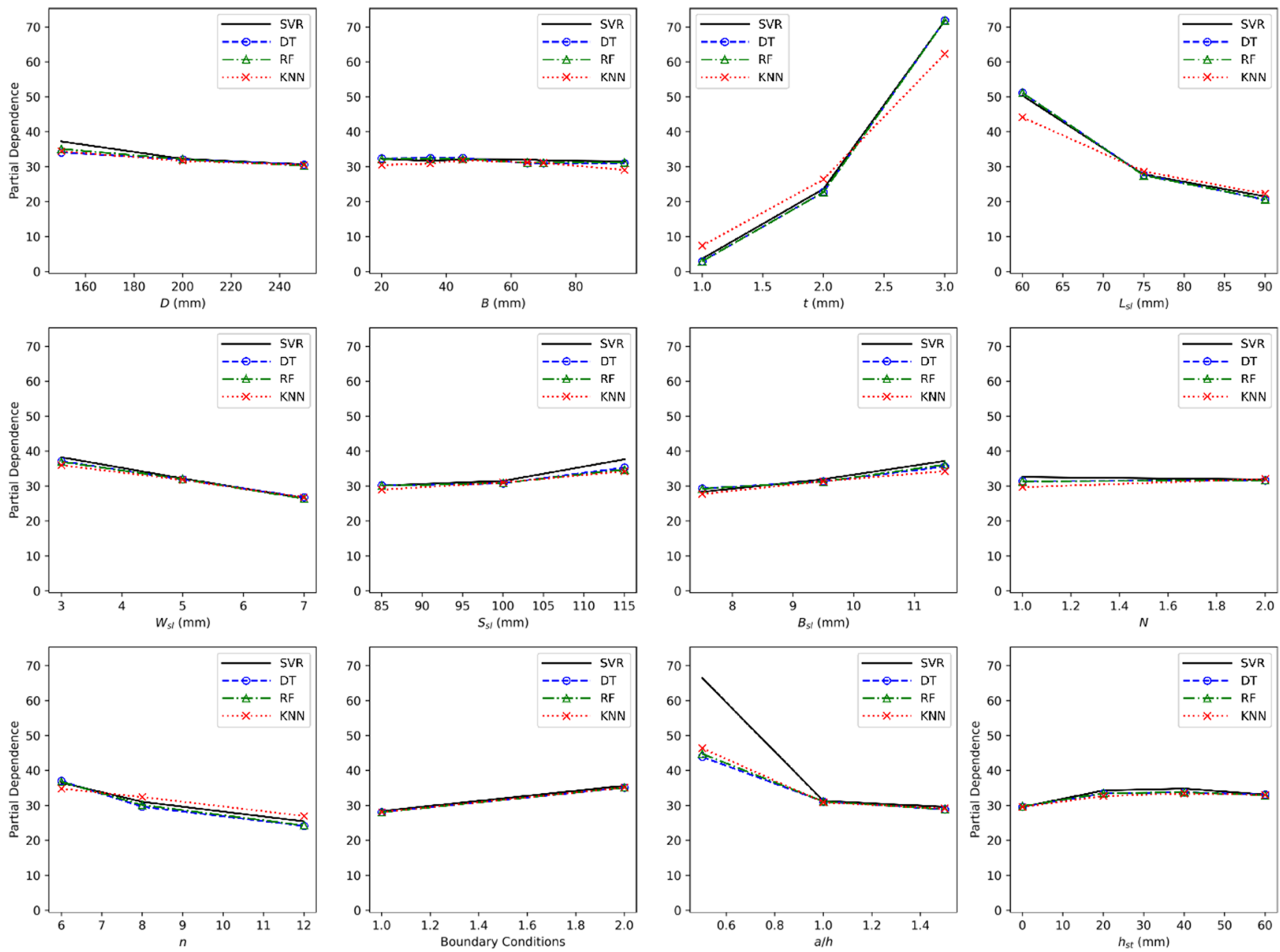

Fig. 11 Partial dependence plots for $V_{c r}$

$N$, whereas $V_{n}$ reduces moderately when $N$ increases from 1 to 2 . An increase in the number of slot rows, $n$, results in practically linear reductions of $V_{c r}$ and $V_{n}$, with $V_{n}$ showing a greater reduction. The CFS channels with the realistic boundary conditions (designated with number 1 in the figures) demonstrate slightly smaller $V_{c r}$ and greater $V_{n}$ than the channels with the test setup boundary conditions. The effects of the aspect ratio $a / h$ on $V_{c r}$ and $V_{n}$ are nonlinear. Both predicted variables reduce considerably when $a / h$ increases from 0.5 to 1 and practically do not change with the further increase in $a / h$ from 1 to 1.5 . The longitudinal web stiffener's introduction results in a slight increase in $V_{c r}$, whereas the increase in the stiffener height, $h_{s t}$, does not affect $V_{c r}$. The $V_{n}$ values are approximately the same for the channels without the longitudinal stiffener and with a $20-\mathrm{mm}$ high longitudinal stiffener. Further increase in the stiffener height results in a nonlinear decrease of $V_{n}$, with with more significant reductions occurring for larger stiffeners. Finally, $V_{n}$ increases when the steel yield stress, $F_{y}$, increases.

\section{Comparisons of $V_{c r}$ and $V_{n}$ predicted by $M L$ models and descriptive equations}

The following descriptive equations for predicting $V_{c r}$ and $V_{n}$ were previously developed from the nonlinear regression analysis of the FE simulation results [14-16].

$$
\begin{aligned}
V_{c r}= & \frac{\pi^{2} E k_{v}}{12\left(1-\mu^{2}\right)\left(\frac{h}{t k_{t}}\right)^{2}} A_{w}=\frac{0.904 E k_{v}}{\left(\frac{h}{t k_{t}}\right)^{2}} A_{w} \\
V_{n}= & V_{y} \text { for } \frac{h}{t k_{t}} \leq b \sqrt{\frac{E k_{v}}{F_{y} k_{f}}} \text { (Shear yielding) } \\
V_{n}= & {\left[1-a\left(\frac{V_{c r}}{V_{y}}\right)^{\alpha}\right]\left(\frac{V_{c r}}{V_{y}}\right)^{\alpha} V_{y} \text { for } \frac{h}{t k_{t}} } \\
& >b \sqrt{\frac{E k_{v}}{F_{y} k_{f}}} \text { (Shear buckling) }
\end{aligned}
$$

where $E$ is modulus of elasticity of CFS; $k_{v}$ is shear buckling coefficient of the whole channel section with flat solid web; 

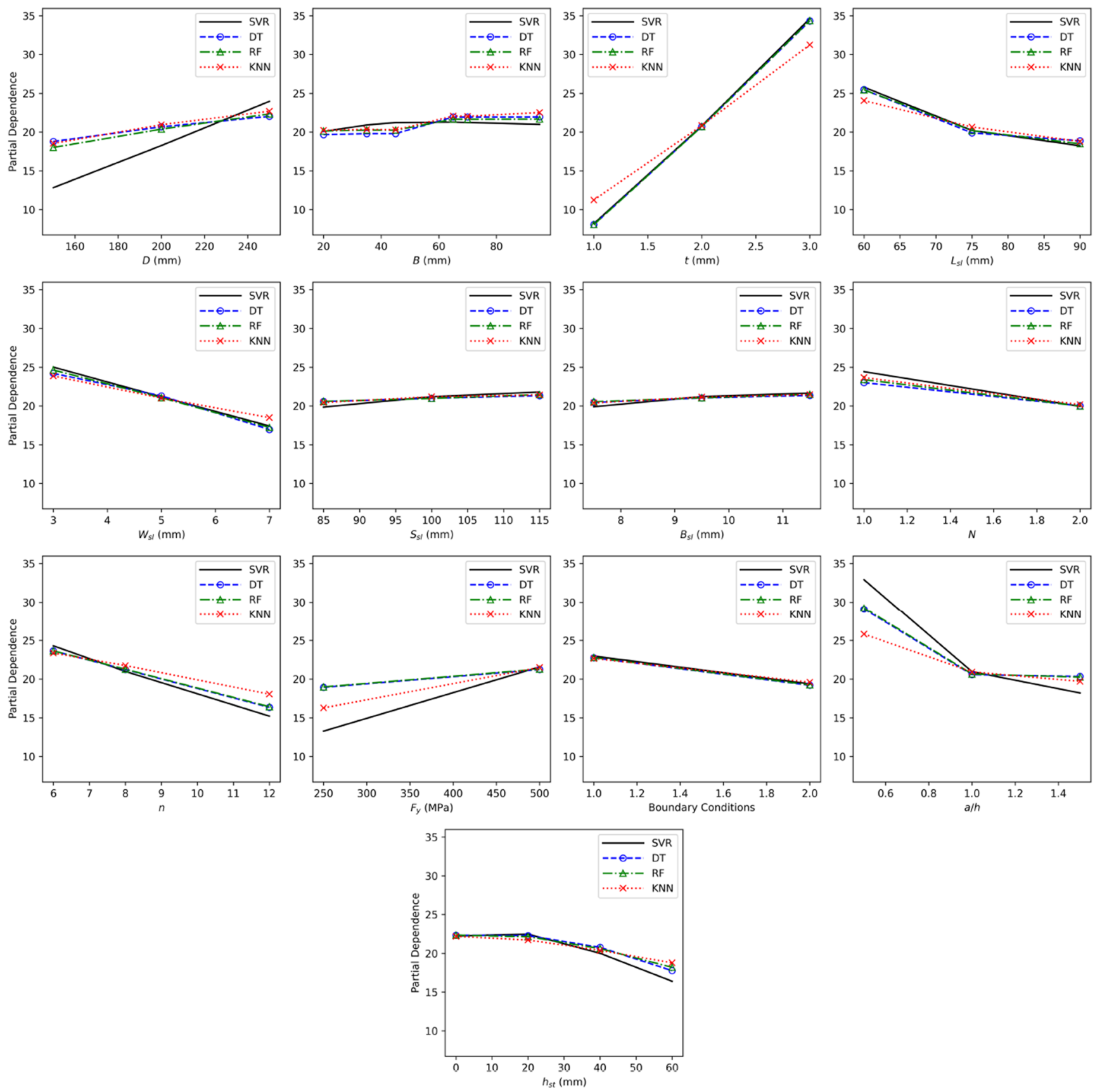

Fig. 12 Partial dependence plots for $V_{n}$

$\mu$ is Poisson's ratio of steel; $h$ is depth of channel web excluding thickness of flanges and rounded corners between the flanges and the web; $t$ is base steel thickness; $A_{w}=h t$ is vertically projected web area irrespective of the stiffener size determined not accounting for web perforations and stiffener; $V_{y}$ is shear yielding strength; $F_{y}$ is yield stress of steel; $k_{t}$ and $k_{f}$ are web thickness modification coefficient and shear strength reduction coefficient due to web perforations determined as described in [14-16]; $a, b$, and $\alpha$ are coefficients ( $a$ is equal to 0.18 and 0.15 for channels with flat and longitudinally stiffened webs, respectively; $b$ is equal to 0.697 and 0.789 for channels with flat and longitudinally stiffened webs, respectively; and $\alpha$ is equal to 0.27 and 0.23 for channels with flat and longitudinally stiffened webs, respectively).

Comparisons of $V_{c r}$ and $V_{n}$ predicted by the developed optimized ML models and the descriptive equations are shown in Figs. 13 and 14. The comparisons demonstrate that SVR, DT, and RF predict $V_{c r}$ and $V_{n}$ from FE simulations with better accuracy than the descriptive equations, whereas 

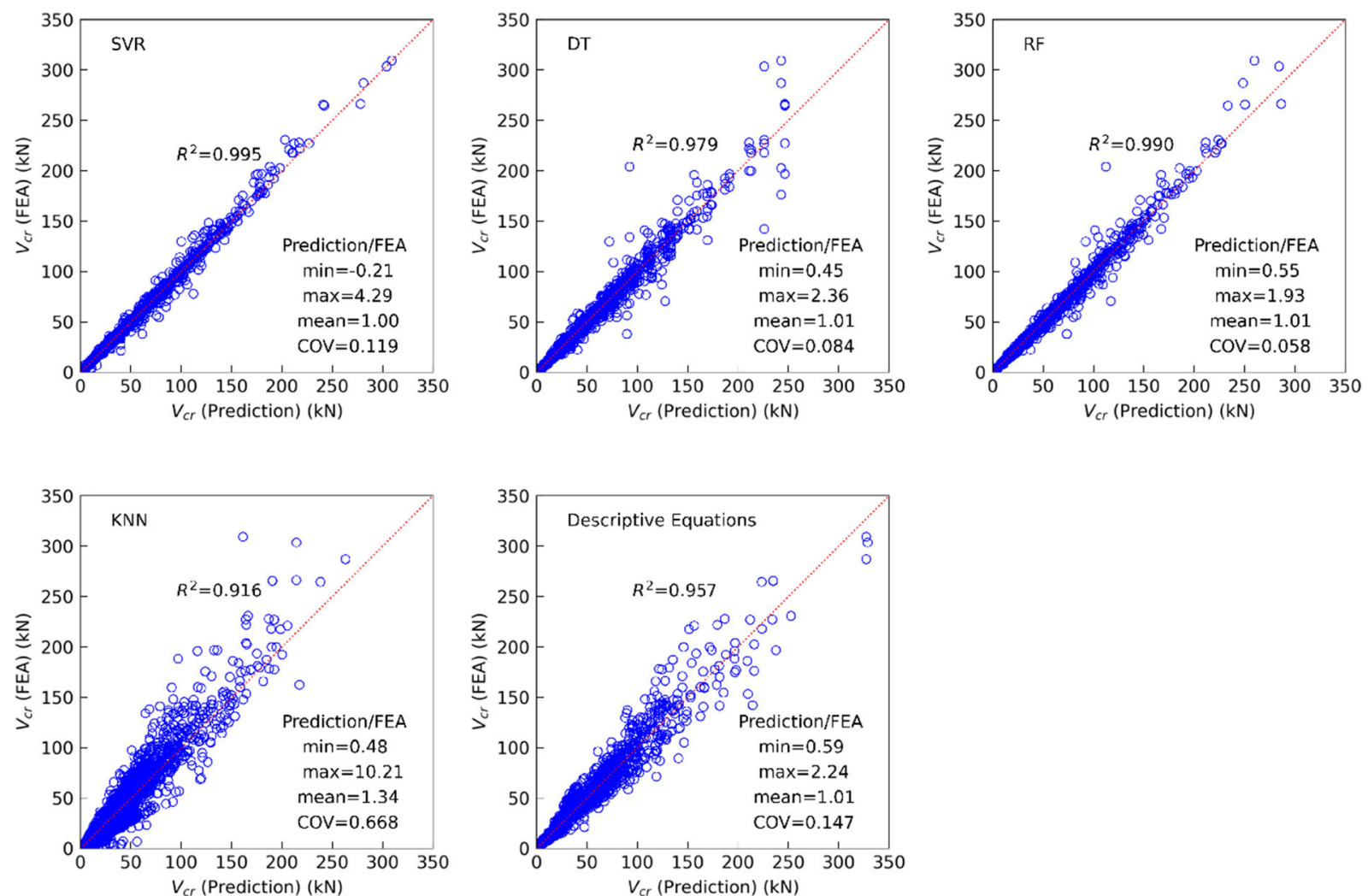

Fig. 13 Comparisons of $V_{c r}$ predicted by the machine learning models and descriptive equations
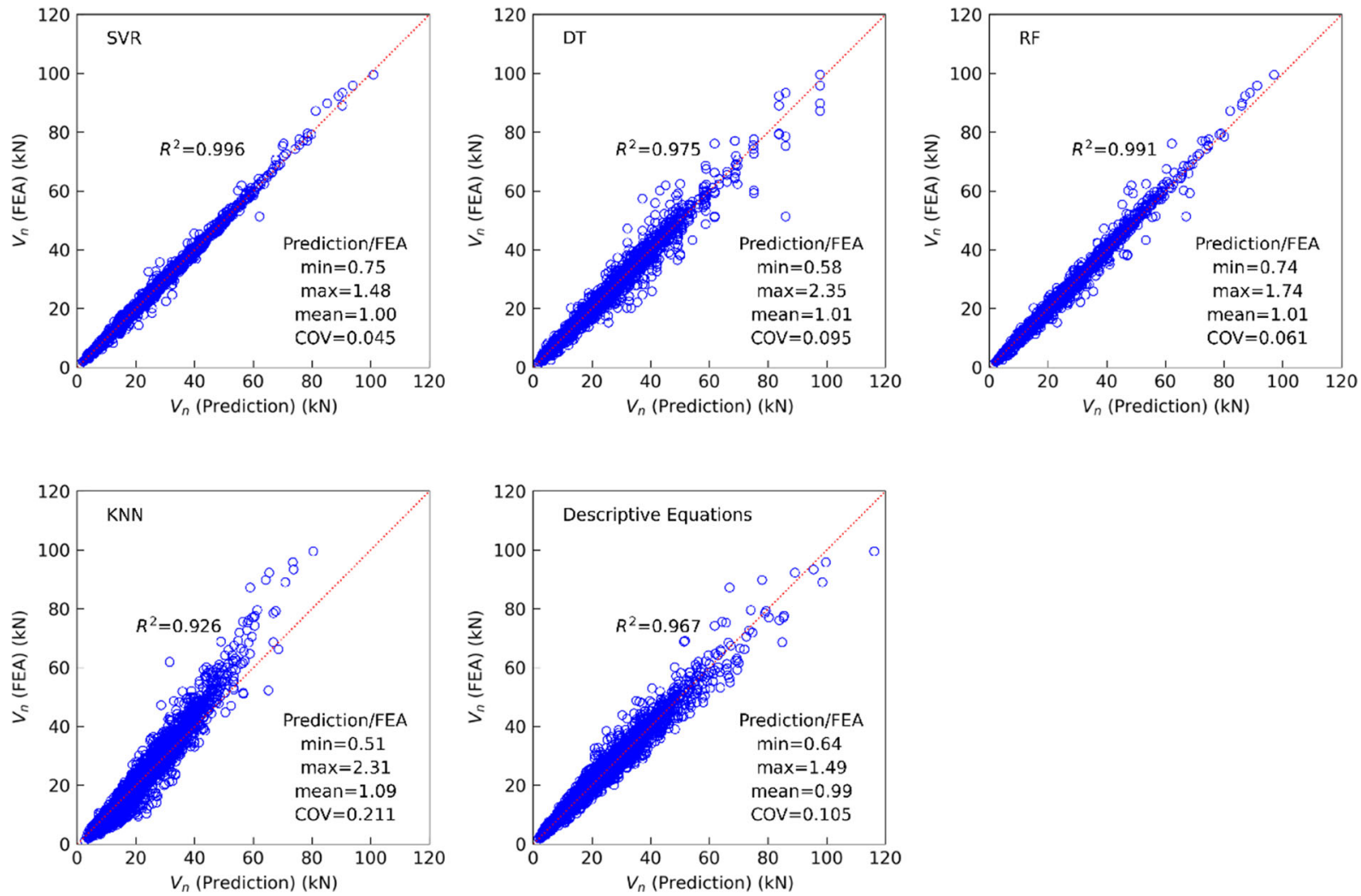

Fig. 14 Comparisons of $V_{n}$ predicted by the machine learning models and descriptive equations 
KNN shows worse prediction abilities. As was mentioned previously, RF and SVR are the best models for predicting $V_{c r}$ and $V_{n}$, respectively.

It is also interesting to compare the performance of the ML models considered in this study with the performance of previously developed ANNs [51]. Tables 1 and 2 present performance metrics for the train, test, and entire sets for SVR, DT, RF, KNN, and ANN models for predicting $V_{c r}$ and $V_{n}$. The model prediction abilities are compared in terms of MSE, MAE, and MAPE for the test set not seen by the models during their training. Table 1 shows that ANNs demonstrate the highest accuracy in predicting $V_{c r}$ than SVR, DT, RF, and KNN. Table 2 shows that the best predictions of $V_{n}$ for all considered performance metrics are provided by SVR, followed by ANNs, RF, DT, and KNN.

\section{Conclusions}

Four machine learning regressors, including support vector machine (SVR), decision tree (DT), random forest (RF), and $\mathrm{k}$-nearest neighbor (KNN), for predicting the elastic shear buckling load, $V_{c r}$, and the ultimate shear strength, $V_{n}$, of CFS channels with slotted webs were developed using a large dataset of FE simulation results with 3512 samples. The models' input parameters were channel depth, channel flange width, channel thickness, length of slots, height of slots, the spacing of slots in the longitudinal and transverse directions, the number of perforated regions, the number of slot rows, type of boundary conditions, the aspect ratio, height of the longitudinal stiffener, and yield stress of steel (in the models for predicting $V_{n}$ only).

The models were validated and tested through tenfold cross-validation, with randomly selected $80 \%$ of the samples used for training and validation and $20 \%$ of the samples used for final testing. The models were finetuned by specifying optimal hyperparameters obtained from an extensive search. The optimal hyperparameters were selected based on the models' performance metrics determined for the test set, which was not used for the model training. Predictions of SVR, DT, and RF models with the optimal hyperparameters compare reasonably well with the FE simulation results, whereas KNN demonstrates considerably worse performance. RF and SVR are the best models, among the ones considered in this study, for predicting $V_{c r}$ and $V_{n}$, respectively. The models were implemented in an open-source Pythonbased framework and allow for a quick and accurate assessment of the elastic shear buckling load and the ultimate shear strength of CFS channels with slotted webs.

The relative feature performance analyses performed for each model using the permutation and SHAP methods allowed for determining the impact of the CFS channel features on the predicted $V_{c r}$ and $V_{n}$ values. It was found that channel thickness, length of slots, and channel boundary conditions have the most significant impact on $V_{c r}$, with channel thickness, channel depth, and the number of slot rows being the most important features for predicting $V_{n}$. The relative importance of channel thickness for predicting $V_{c r}$ and $V_{n}$ significantly exceeds the importance of other features. Partial dependence analysis allowed for obtaining partial dependence plots, demonstrating how $V_{c r}$ and $V_{n}$ change with the change of each feature.

$V_{c r}$ and $V_{n}$ predicted by the developed ML models were compared with the predictions by the existing descriptive equations and previously developed ANNs. SVR, DT, and RF demonstrate superior performance compared with the descriptive equations. Among the ML models, ANNs show the best performance in predicting $V_{c r}$, whereas SVR is the best model for predicting $V_{n}$.

\section{References}

1. Höglund T, Burstrand H (1998) Slotted steel studs to reduce thermal bridges in insulated walls. Thin-Walled Struct 32(13):81-109. https://doi.org/10.1016/S0263-8231(98)00028-7

2. Lipták-Váradi J (2010) Equivalent thermal conductivity of steel girders with slotted web. Periodica Polytechnica Civil Engineering 54(22):163-170. https://doi.org/10.3311/pp.ci.2010-2.12

3. Kesti J (2000) Local and distortional buckling of perforated steel wall studs. PhD thesis, Helsinki University of Technology

4. Salhab B, Wang YC (2008) Equivalent thickness of cold-formed thin-walled channel sections with perforated webs under compression. Thin-Walled Struct 46(7):823-838. https://doi.org/10.1016/ j.tws.2008.01.029

5. Kwon YB, Soe EK, Lim DM, Kim GD, Kwon IK (2012) The compressive strength of thin-walled cold-formed steel studs with slits in the web. Journal of Korean Society of Steel Construction 24(2):189-197. https://doi.org/10.7781/kjoss.2012.24.2.189

6. Kwon YB, Kim GD, Kwon IK (2014) Compression tests of coldformed channel sections with perforations in the web. Steel and Composite Structures 16(6):657-679. https://doi.org/10.12989/ scs.2014.16.6.657

7. Naser MZ, Degtyareva NV (2019) Temperature-induced instability in cold-formed steel beams with slotted webs subject to shear. Thin-Walled Struct 136:333-352. https://doi.org/10.1016/j.tws. 2018.12.030

8. Visy D, Szedlák M, Geleji BB, Ádány S (2019) Flexural buckling of thin-walled lipped channel columns with slotted webs: numerical and analytical studies. Eng Struct 197:109399. https://doi.org/10.1016/j.engstruct.2019.109399

9. Degtyareva N, Gatheeshgar P, Poologanathan K, Gunalan S, Lawson M, Sunday P (2019) Combined bending and shear behaviour of slotted perforated steel channels: numerical studies. J Constr Steel Res 161:369-384. https://doi.org/10.1016/j.jcsr.2019.07.008

10. Degtyareva N, Gatheeshgar P, Poologanathan K, Gunalan S, Shyha I, McIntosh A (2020) Local buckling strength and design of cold-formed steel beams with slotted perforations. Thin-Walled Struct 156:106951. https://doi.org/10.1016/j.tws.2020.106951

11. Degtyareva N, Gatheeshgar P, Poologanathan K, Gunalan S, Tsavdaridis KD, Napper S (2020) New distortional buckling design 
rules for slotted perforated cold-formed steel beams. J Constr Steel Res 168:106006. https://doi.org/10.1016/j.jcsr.2020.106006

12. Degtyareva NV, Degtyarev VV (2016) Experimental investigation of cold-formed steel channels with slotted webs in shear. ThinWalled Struct 102:30-42. https://doi.org/10.1016/j.tws.2016.01. 012

13. Degtyareva N, Poologanathan K, Mahendran M (2021) Web crippling tests of cold-formed steel channels with staggered web perforations. Thin-Walled Struct 159:107314. https://doi.org/10. 1016/j.tws.2020.107314

14. Degtyarev VV, Degtyareva NV (2017) Numerical simulations on cold-formed steel channels with flat slotted webs in shear. Part I: elastic shear buckling characteristics. Thin-Walled Struct 119:22-32. https://doi.org/10.1016/j.tws.2017.05.026

15. Degtyarev VV, Degtyareva NV (2017) Numerical simulations on cold-formed steel channels with flat slotted webs in shear. Part II: ultimate shear strength. Thin-Walled Struct 119:211-223. https://doi.org/10.1016/j.tws.2017.05.028

16. Degtyarev VV, Degtyareva NV (2018) Numerical simulations on cold-formed steel channels with longitudinally stiffened slotted webs in shear. Thin-Walled Struct 129:429-456. https://doi.org/10.1016/j.tws.2018.05.001

17. Degtyarev VV, Degtyareva NV (2016) Finite element modeling of cold-formed steel channels with solid and slotted webs in shear. Thin-Walled Struct 103:183-198. https://doi.org/10.1016/j.tws. 2016.02.016

18. Degtyarev V, Degtyareva N (2020) Elastic shear buckling loads and ultimate shear strengths of CFS channels with slotted webs: FE simulation results. Mendeley Data. https://doi.org/10.17632/ t9hhkngdwv.1

19. Jiang F, Jiang Y, Zhi H, Dong Y, Li H, Ma S, Wang Y, Dong Q, Shen H, Wang Y (2017) Artificial intelligence in healthcare: past, present and future. Stroke and Vascular Neurology 2(4). https://doi.org/10.1136/svn-2017-000101

20. Tadapaneni NR (2019) Artificial intelligence in finance and investments. Int J Innovative Res Sci Eng Technol 9(5):2792-2795

21. Ma Y, Wang Z, Yang H, Yang L (2020) Artificial intelligence applications in the development of autonomous vehicles: a survey. IEEE/CAA Journal of Automatica Sinica 7(2):315-329. https://doi.org/10.1109/JAS.2020.1003021

22. Wuest T, Weimer D, Irgens C, Thoben KD (2016) Machine learning in manufacturing: advantages, challenges, and applications. Production \& Manufacturing Research 4(1):23-45. https://doi.org/10.1080/21693277.2016.1192517

23. Kartam N, Flood I, Garrett JH (1997) Artificial neural networks for civil engineers: fundamentals and applications. American Society of Civil Engineers. New York, NY

24. Adeli H (2001) Neural networks in civil engineering: 1989-2000. Computer-Aided Civil and Infrastructure Engineering 16(2):126142. https://doi.org/10.1111/0885-9507.00219

25. Salehi H, Burgueño R (2018) Emerging artificial intelligence methods in structural engineering. Eng Struct 171:170-189. https://doi.org/10.1016/j.engstruct.2018.05.084

26. Naser MZ (2021) Systematic Integration of Artificial Intelligence Toward Evaluating Response of Materials and Structures in Extreme Conditions. In: Deo R., Samui P., Kisi O., Yaseen Z. (eds) Intelligent Data Analytics for Decision-Support Systems in Hazard Mitigation. Springer Transactions in Civil and Environmental Engineering. Springer, Singapore. https://doi.org/ 10.1007/978-981-15-5772-9_10

27. Naser MZ (2021) Mechanistically informed machine learning and artificial intelligence in fire engineering and sciences. Fire Technol. https://doi.org/10.1007/s10694-020-01069-8

28. Kumar M, Yadav N (2013) Buckling analysis of a beam-column using multilayer perceptron neural network technique. J Frankl
Inst 350(10):3188-3204. https://doi.org/10.1016/j.jfranklin.2013. 07.016

29. Abambres M, Rajana K, Tsavdaridis KD, Ribeiro TP (2019) Neural network-based formula for the buckling load prediction of I-section cellular steel beams. Computers 8(1):2-26. https://doi.org/10.3390/computers 8010002

30. Tashakori A, Adeli H (2002) Optimum design of cold-formed steel space structures using neural dynamics model. J Constr Steel Res 58(12):1545-1566. https://doi.org/10.1016/S0143-974X(01)001 $05-5$

31. Guzelbey IH, Cevik A, Erklig A (2006) Prediction of web crippling strength of cold-formed steel sheetings using neural networks. J Constr Steel Res 62(10):962-973. https://doi.org/10. 1016/j.jcsr.2006.01.008

32. Pala M (2006) A new formulation for distortional buckling stress in cold-formed steel members. J Constr Steel Res 62(7):716-722. https://doi.org/10.1016/j.jcsr.2005.09.011

33. Pala M, Caglar N (2007) A parametric study for distortional buckling stress on cold-formed steel using a neural network. J Constr Steel Res 63(5):686-691. https://doi.org/10.1016/j.jcsr.2006.07. 005

34. D’Aniello M, Güneyisi EM, Landolfo R, Mermerdaş K (2014) Analytical prediction of available rotation capacity of cold-formed rectangular and square hollow section beams. Thin-Walled Struct 77:141-152. https://doi.org/10.1016/j.tws.2013.09.015

35. Çevik A, Kurtoğlu AE, Bilgehan M, Gülşan ME, Albegmprli HM (2015) Support vector machines in structural engineering: a review. Journal of Civil Engineering and Management 21(3):261281. https://doi.org/10.3846/13923730.2015.1005021

36. Liu C, Liu C, Liu C, Huang X, Miao J, Xu W (2019) Fire damage identification in $\mathrm{RC}$ beams based on support vector machines considering vibration test. KSCE J Civ Eng 23(10):4407-4416. https://doi.org/10.1007/s12205-019-2353-7

37. Solhmirzaei R, Salehi H, Kodur V, Naser M (2020) Machine learning framework for predicting failure mode and shear capacity of ultra high performance concrete beams. Eng Struct 224:111221. https://doi.org/10.1016/j.engstruct.2020.111221

38. Ahmad MS, Adnan SM, Zaidi S, Bhargava P (2020) A novel support vector regression (SVR) model for the prediction of splice strength of the unconfined beam specimens. Constr Build Mater 248:118475. https://doi.org/10.1016/j.conbuildmat.2020.118 475

39. Nguyen H, Vu T, Vo TP, Thai HT (2021) Efficient machine learning models for prediction of concrete strengths. Constr Build Mater 266:120950. https://doi.org/10.1016/j.conbuildmat.2020. 120950

40. Kang MC, Yoo DY, Gupta R (2021) Machine learningbased prediction for compressive and flexural strengths of steel fiber-reinforced concrete. Constr Build Mater 266:121117. https://doi.org/10.1016/j.conbuildmat.2020.121117

41. Su M, Zhong Q, Peng H, Li S (2021) Selected machine learning approaches for predicting the interfacial bond strength between FRPs and concrete. Constr Build Mater 270:121456. https://doi.org/10.1016/j.conbuildmat.2020.121456

42. Mangalathu S, Jang H, Hwang SH, Jeon JS (2020) Datadriven machine-learning-based seismic failure mode identification of reinforced concrete shear walls. Eng Struct 208:110331. https://doi.org/10.1016/j.engstruct.2020.110331

43. Zhang J, Ma G, Huang Y, Aslani F, Nener B et al (2019) Modelling uniaxial compressive strength of lightweight selfcompacting concrete using random forest regression. Constr Build Mater 210:713-719. https://doi.org/10.1016/j.conbuildmat.2019. 03.189

44. Mangalathu S, Hwang SH, Jeon JS (2020) Failure mode and effects analysis of RC members based on machine-learning-based 
SHapley Additive exPlanations (SHAP) approach. Eng Struct 219:110927. https://doi.org/10.1016/j.engstruct.2020.110927

45. Olalusi OB, Awoyera PO (2021) Shear capacity prediction of slender reinforced concrete structures with steel fibers using machine learning. Eng Struct 227:111470. https://doi.org/10.1016/j.eng struct.2020.111470

46. Naser M, Salehi H (2020) Machine learning-driven assessment of fire-induced concrete spalling of columns. ACI Mater J 117(6):716. https://doi.org/10.14359/51728120

47. Lyu ZJ, Zhao P, Lu Q, Xiang Q, Li H (2020) Prediction of the bending strength of boltless steel connections in storage pallet racks: an integrated experimental-FEM-SVM methodology. Advances in Civil Engineering 2020:5109204. https://doi.org/10.1155/2020/5109204

48. Kim SH, Song X, Cho C, Lee CH (2021) Strength prediction of steel CHS X-joints via leveraging finite element method and machine learning solutions. J Constr Steel Res 176:106394. https://doi.org/10.1016/j.jcsr.2020.106394

49. Panev Y, Kotsovinos P, Deeny S, Flint G (2021) The use of machine learning for the prediction of fire resistance of composite shallow floor systems. Fire Technol. https://doi.org/10. 1007/s10694-021-01108-y

50. Fu F (2020) Fire induced progressive collapse potential assessment of steel framed buildings using machine learning. J Constr Steel Res 166:105918. https://doi.org/10.1016/j.jcsr.2019.105918

51. Degtyarev VV (2021) Neural networks for predicting shear strength of CFS channels with slotted webs. J Constr Steel Res 177:106443. https://doi.org/10.1016/j.jcsr.2020.106443

52. Hastie T, Tibshirani R, Friedman J (2009) The elements of statistical learning: data mining, inference, and prediction. Springer Science \& Business Media

53. Géron A (2019) Hands-on machine learning with Scikit-Learn, Keras, and TensorFlow: concepts, tools, and techniques to build intelligent systems. O'Reilly Media
54. Vapnik V (1995) The nature of statistical learning theory. Springer, New York

55. Cortes C, Vapnik V (1995) Support-vector networks. Machine Learning 20(3):273-297

56. Vapnik V, Golowich SE, Smola A et al (1997) Support vector method for function approximation, regression estimation, and signal processing. Advances in Neural Information Processing Systems, pp 281-287

57. Smola AJ, Schölkopf B (2004) A tutorial on support vector regression. Statistics and Computing 14(3):199-222

58. Pedregosa F, Varoquaux G, Gramfort A, Michel V, Thirion B, Grisel O, Blondel M, Prettenhofer P, Weiss R, Dubourg V et al (2011) Scikit-learn: machine learning in python. The Journal of Machine Learning Research 12:2825-2830

59. Breiman L (2001) Random forests. Machine Learning 45(1):5-32

60. Ho TK (1995) Random decision forests. In: Proceedings of 3rd international conference on document analysis and recognition, vol 1. IEEE, pp 278-282

61. Claesen M, Simm J, Popovic D, Moreau Y, De Moor B (2014) Easy hyperparameter search using optunity. arXiv:14121114

62. Clerc M, Kennedy J (2002) The particle swarm-explosion, stability, and convergence in a multidimensional complex space. IEEE Transactions on Evolutionary Computation 6(1):58-73

63. Naser M, Alavi A (2020) Insights into performance fitness and error metrics for machine learning. arXiv:200600887

64. Lundberg S, Lee SI (2017) A unified approach to interpreting model predictions. arXiv:170507874

Publisher's Note Springer Nature remains neutral with regard to jurisdictional claims in published maps and institutional affiliations. 